Published in final edited form as:

Nature. 2018 May ; 557(7703): 123-126. doi:10.1038/s41586-018-0061-y.

\title{
Structure of the Alternative Complex III in a supercomplex with cytochrome oxidase
}

\author{
Chang Sun ${ }^{1,2, a}$, Samir Benlekbir ${ }^{3, a}$, Padmaja Venkatakrishnan ${ }^{1, a, b}$, Yuhang Wang ${ }^{1,2,4}$,

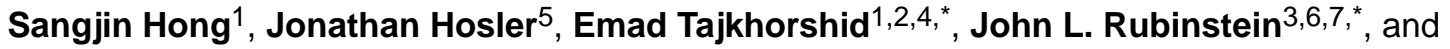 \\ Robert B. Gennis ${ }^{1, *}$ \\ ${ }^{1}$ Department of Biochemistry, University of Illinois, 600 S. Mathews Street, Urbana, IL 61801, \\ USA
}

${ }^{2} \mathrm{NIH}$ Center for Macromolecular Modeling and Bioinformatics, Beckman Institute for Advanced Science and Technology, University of Illinois, 405 N. Mathews, Urbana, IL 61801, USA

${ }^{3}$ Molecular Medicine Program, The Hospital for Sick Children Research Institute, 686 Bay Street, Toronto, ON M5G 0A4, Canada

${ }^{4}$ Center for Biophysics and Quantitative Biology, University of Illinois, 1110 Green Street, Urbana, IL 61801, USA

${ }^{5}$ Department of Biochemistry, University of Mississippi Medical Center, 2500 N. State Street, Jackson, MS 39216, USA

${ }^{6}$ Department of Medical Biophysics, The University of Toronto, Suite 15-701, 101 College Street Toronto, ON M5G 1L7, Canada

${ }^{7}$ Department of Biochemistry, The University of Toronto, Room 5207, 1 King's College Circle, Toronto, ON M5S 1A8, Canada

\begin{abstract}
Alternative Complex III (ACIII) is a key component of the respiratory and/or photosynthetic electron transport chains of many bacteria ${ }^{1-3}$. Like Complex III ( $b c_{1}$ complex), ACIII catalyzes the oxidation of membrane-bound quinol and reduction of cytochrome $c$ or an equivalent electron carrier. However, the two complexes have no structural similarity ${ }^{4-7}$.
\end{abstract}

\footnotetext{
Users may view, print, copy, and download text and data-mine the content in such documents, for the purposes of academic research, subject always to the full Conditions of use: http://www.nature.com/authors/editorial_policies/license.html\#termsInformation for reprints and permissions is available at www.nature.com/reprints.

"Co-corresponding authors: J.L.R.: john.rubinstein@utoronto.ca, TEL: 416-813-7255, R.B.G.: r-gennis@illinois.edu, TEL: 217-333-9075, E.T.: emad@ life.illinois.edu, TEL: 217-244-6941.

${ }^{a}$ Chang Sun, Samir Benlekbir and Padmaja Venkatakrishnan contributed equally to the work in this manuscript

bCurrent address: Department of Microbiology and Molecular Genetics, UC Davis, One Shields Avenue, Davis, CA 95616

Author Contributions: P.V. expressed, purified and characterized the protein supercomplex, S.B. prepared cryo-EM specimens and calculated cryo-EM maps, C.S. built the de novo atomic structures, Y.W. conducted the molecular dynamics simulations, S.H. performed the electrochemical characterization, J.H. performed the metal analysis, E.T. supervised molecular dynamics simulations, J.L.R supervised the molecular structure determination, and R.B.G. conceived the project, supervised biochemical experiments, and coordinated the project. All authors participated in the manuscript preparation.

The cryo-EM density maps have been deposited in the Electron Microscopy Data Bank under accessions EMD-7286, EMD-7447, EMD-7448, the atomic coordinates for ACIII have been deposited in the Protein Data Bank under accession 6BTM.

The authors declare no competing financial interests.

Readers are welcome to comment on the online version of the paper.
} 
Although ACIII has eluded structural characterization, several of its subunits are homologous to members of the CISM (Complex Iron-Sulfur Molybdoenzyme) superfamily ${ }^{8}$, including the proton pump polysulfide reductase ${ }^{9,10}$. We isolated the ACIII from Flavobacterium johnsoniae with native lipids using styrene maleic acid (SMA) copolymer $^{11-14}$, both as an independent enzyme and as a functional 1:1 supercomplex with an $a a_{3}$-type cytochrome $c$ oxidase (cyt $a a_{3}$ ). We determined the structure of ACIII to $3.4 \AA$ resolution by cryo-EM and constructed an atomic model for its six subunits. The structure, which contains a [3Fe-4S] cluster, a [4Fe-4S] cluster, and six hemes $c$, shows that ACIII employs known elements from other electron transport complexes arranged in a previously unknown manner. Modeling of the cyt aa 3 component of the supercomplex revealed that it is structurally modified to facilitate association with ACIII, illustrating the importance of the supercomplex in this electron transport chain. The structure also resolves two of the subunits of ACIII that are anchored to the lipid bilayer with $\mathrm{N}$-terminal triacylated cysteine residues, an important post-translational modification found in numerous prokaryotic membrane proteins that has not previously been observed structurally in a lipid bilayer.

The ACIII-cyt $a a_{3}$ supercomplex from $F$. johnsoniae membranes was solubilized, purified, and biochemically characterized using styrene maleic acid (SMA) copolymer nanodiscs without traditional detergents (Supplementary Discussion, Extended Data Fig. 1-3). The supercomplex catalyzes the two-electron oxidiation of menaquinol (or ubiquinol) and the four-electron reduction of $\mathrm{O}_{2}$ to water with a turnover number of $\sim 21$ electrons/sec without the addition of exogenous cyt $c$ (Supplementary Information, Extended Data Fig. 3), indicating a functional electron transfer chain within the supercomplex. The addition of exogeneous cyt $c$ did not increase the rate of electron transfer. The structure of the ACIII-cyt $a a_{3}$ supercomplex in SMA nanodiscs was determined by cryo-EM (Fig. 1, Extended Data Fig. 4). The supercomplex has a mass of $464 \mathrm{kDa}$ (Supplementary Discussion), a transmembrane cross-section of $\sim 9 \mathrm{~nm} \times 13 \mathrm{~nm}$ (Extended Data Fig. 5), and contains 48 transmembrane a-helices. To our knowledge, the ACIII-aaz supercomplex is the largest protein complex reported to be contained within an SMA copolymer nanodisc. The SMA copolymer and lipids contribute only a thin layer of density around the supercomplex (Fig. $1 \mathrm{a}$ and $1 \mathrm{~b}$ ), which is not circular but follows the contours of the protein. Whether this is a general feature of SMA-solubilized proteins or is due to the large size of the ACIII-aa 3 supercomplex is not known, and will be clarified when more structures are determined using this approach. The number of loosely bound, unresolved lipid molecules is not known, nor whether they are in sufficient number to form a true bilayer surrounding the protein. The SMA-supercomplex nanodiscs retain native lipids, are more stable, and have $30 \%$ higher specific activity than the supercomplex isolated with detergents (e.g., dodecylmaltoside) (Supplementary Discussion, Extended Data Fig. 3). Since traditional detergents are avoided in generating SMA nanodiscs, the preparative protocol is relatively rapid and simpler than making nanodiscs using the membrane scaffold protein (MSP).

While the properties of the SMA nanodiscs are less well characterized than nanodiscs made with membrane scaffolding protein ${ }^{13,15}$, our work demonstrates the utility of SMA nanodiscs for high-resolution structural studies of membrane proteins. 
The resolution of the cryo-EM density map allowed construction of an atomic model for > 90\% of the sequences predicted from the ACIII gene cluster (Supplementary Discussion), including subunits ActA, ActB, ActC, ActD, ActE, and ActF (Fig. 2 and Extended Data Fig. 5, Extended Data Table 1). Sequence analysis shows that ACIII contains a unique combination of known modules from other respiratory complexes ${ }^{3}$ (Supplementary Discussion). The ACIII structure confirms this prediction and shows the structure responsible catalyzing the quinol:cytochrome $c$ oxidoreductase activity. The ACIII structure can be divided into three parts: (i) a core assembly of ActC and ActB that oxidizes quinol; (ii) A heme $c$ assembly consisting of ActA and ActE that directs electron from ActB to the terminal electron acceptor; and (iii) Auxiliary transmembrane subunits ActD and ActF with unknown functions. With some key differences (Extended Data Fig. 5), the overall architecture of ActB and ActC resembles the PsrA/PsrB and PsrC subunits of polysulfide reductase from Thermus thermophilus (PsrABC) ${ }^{16}$, a member of the CISM superfamily (Supplementary Discussion). Like PsrC, ActC contains no cofactors but does contain the proposed site for menaquinol oxidation. Residues at the menaquinol binding site identified in $\mathrm{PsrC}^{16}$ are not conserved in $\mathrm{ActC}^{17}$. Although menaquinone is not observed in the cryoEM map, we propose that His $133^{\mathrm{C}}$ and $\mathrm{Asp} 164^{\mathrm{C}}$ form the menaquinol binding site in ActC near the interface with ActB (Extended Data Fig. 6). These two residues are conserved in ActC sequences and there is a crevice between TM3 and TM4 of ActC that would provide access to the substrate in the membrane bilayer.

The N-terminal portion of ActB is homologous to the PsrA subunit of polysulfide reductase, which contains the molybdenum cofactor, but the molybdenum cofactor is absent in $\mathrm{ActB}^{4}$. The C-terminal domain of ActB is homologous to PsrB and both ActB and PsrB contain Fe$\mathrm{S}$ clusters. Like PsrB, ActB from F. johnsoniae is expected to contain 4 [Fe-S] clusters, but only two are observed in the cryo-EM map (Extended Data Fig. 7). There is one [3Fe-4S] cluster near the interface with ActC, about $10 \AA$ from the proposed site of menaquinol oxidation, and one $[4 \mathrm{Fe}-4 \mathrm{~S}]$ cluster about $9 \AA$ further away. There are two additional cysteine clusters present in the structure of ActB, but the cryo-EM map does not show [FeS] clusters at these locations. Instead, we observe disulfide bonds (Cys965 $/$ Cys $938^{\mathrm{B}}$ and Cys $971^{\mathrm{B}} / \mathrm{Cys} 769^{\mathrm{B}}$ ) within these two cysteine clusters. Substitution of proposed $[4 \mathrm{Fe}-4 \mathrm{~S}]$ clusters by disulfide bonds may be a genuine aspect of the structure or may be due to oxidation that occurred during sample preparation. However, if these two "missing" [4Fe-4S] clusters were present, they would form a dead-end for electron transfer from the $[3 \mathrm{Fe}-4 \mathrm{~S}]$ cluster of ActB suggesting that their absence from the structure is not an artifact.

The [3Fe-4S] cluster in ActB is the most probable initial oxidant of menaquinol bound to ActC, and is $12.3 \AA$ from the nearest heme $c$ in ActA. The five hemes $c$ in ActA plus the single heme $c$ in ActE form a likely electron transfer wire from the $[3 \mathrm{Fe}-4 \mathrm{~S}]$ cluster in ActB, with the largest edge-to-edge distance of $9.2 \AA$ between adjacent hemes (Fig. 2b). The [4Fe-4S] cluster in ActB appears to be off-pathway and its function remains to be determined.

In all Flavobacteria, including $F$. johnsoniae, ActA is predicted to have a monoheme domain at the $\mathrm{N}$ terminus in addition to the pentaheme domain at the $\mathrm{C}$ terminus (Supplementary Discussion). Mass spectrometry analysis shows that the $\mathrm{N}$-terminal monoheme domain is 
present in the preparation (Extended Data Fig. 1), but no density can be assigned to this entire domain. The inability to resolve the monoheme domain may result from flexibility of the domain. Full-atom molecular dynamics (MD) simulations were performed for the entire structure of ACIII embedded in a phospholipid bilayer to determine the stability and dynamics of the structure (Extended Data Fig. 8). Interestingly, the pentaheme domain of ActA had the largest root-mean-square deviation (RMSD), which is mainly from the transmembrane a-helix connected to the missing monoheme domain, consistent with the monoheme domain being unobservable due to a variable position in the complex. While ActE also had a significant RMSD, that did not appear to correlate with disorder in the cryoEM map.

ActD and ActF are transmembrane subunits without bound cofactors, and both interact with ActC. It has not been established if ACIII generates a proton motive force coupled to electron transport ${ }^{18}$. The absence of redox centers in ActC, ActD and ActF suggests that if ACIII contributes to the transmembrane proton gradient, it does not use the bifurcation-type Q cycle mechanism of canonical Complex III $^{19}$, but instead uses a true proton pump mechanism like Complex $\mathrm{I}^{20}$. ActD has two transmembrane a-helices that cross within the membrane and are adjacent to ActC. Both $\mathrm{N}$ - and C-termini are within the cytoplasm and combine to form a single globular domain that rests on the cytoplasmic surface of ActC. The ten transmembrane a-helices of ActF form a pseudo two-fold axis of symmetry with the ten transmembrane a-helices of ActC (Extended Data Fig. 5) despite the fact that ActF has less than $20 \%$ sequence identity with ActC. If ACIII is a proton pump, it is likely that conserved polar residues within the bilayer will play important roles.

The structure of ACIII reveals eleven ordered phospholipid molecules as well as triacylated cysteine residues at the $\mathrm{N}$ termini of ActB (Fig. 3a) and ActE (Extended Data Fig. 7). Anchoring of bacterial membrane proteins by an N-terminal triacylated cysteine is a well characterized phenomenon ${ }^{21}$ but, to our knowledge, this is the first time the structure of a triacylated cysteine residue has been determined in the context of a membrane protein. Surprisingly, both lipid anchors are tilted with respect to the plane of the lipid bilayer (Fig. 2a), restricting the ability of other lipids to pack around them. This feature could alter the mechanical properties of the adjacent portion of the membrane bilayer and also guide conformational changes in the ACIII protein. Remarkably, the two N-terminal lipid anchors are adjacent to each other in the membrane. These lipid anchors likely help ACIII to assemble and keep the monoheme ActE bound to the complex. The eleven lipids that are resolved adjacent to the transmembrane $a$-helices accommodate the rugged protein surface of the complex (Fig. 3b and Extended Data Fig. 7). The headgroups of the lipids could not be identified and they were all modeled as phosphatidylethanolamine. There are two "hot spots" for resolved lipids: (i) The cytoplasmic interface between ActC and ActF; and (ii) The vicinity of the triacylated cysteine of $\mathrm{ActB}$, which is near the proposed entry point for menaquinol into the complex. All eleven of the resolved lipids remained bound to the protein throughout $250 \mathrm{~ns}$ of MD simulation (Extended Data Fig. 8), supporting the ability of SMA-nanodiscs to preserve some native lipid-protein interactions and suggesting a functional role for the lipids. A large number of annular lipids, including those modelled in the structure, were observed to associate with the protein from the in silico bilayer. 
Frequently, the subunits encoding ACIII are within an operon that includes subunits of an associated Complex IV ${ }^{3}$ (cyt $a a_{3}$ or cyt $c a a_{3}$ ). We find that the sequences of subunit III from Complex IVs that are associated with ACIII have unusual features that distinguish them from the canonical subunit III (Supplementary Discussion). Whereas subunit III of Complex IV generally contains seven transmembrane a-helices, those that are associated with ACIII lack TM1 and TM2 (Fig. 4a). While only parts of subunit III of cyt $a a_{3}$ are resolved at better than $4 \AA$, the density for cyt $a a_{3}$ has sufficient resolution to identify five a-helices from the structure. A homology model of subunit III from $F$. johnsoniae cyt aa 3 was built based on the structure of TM3 to TM7 of subunit III from Rhodobacter sphaeroides cyt aa 3 and fit into the ACIII-cyt $a a_{3}$ supercomplex density map (Extended Data Fig. 9) with high fidelity. The deletion of the first two transmembrane a-helices in subunit III of cyt $a a_{3}$ appears to be a necessary adaptation to allow formation of the supercomplex with ACIII. It is interesting that the same two helices in subunit III are also absent in the cyt a $a_{3}$ obligatory bcc-cyt $a_{3}$ supercomplex found in Actinobacteria (e.g., Corynebacterium glutamicam and Mycobacterium tuberculosis) ${ }^{22}$

The sequence analysis also reveals that the loop between TM5 and TM6 of subunit III in the cyt $a_{3}$ that is part of the supercomplex is much longer in F. johnsoniae (and all

Flavobacteria) than in other organisms. Typically, this loop contains eight residues, but in $F$. johnsoniae, it contains 121 residues (Fig. 4a). Part of this long loop fits in a groove between ActB and ActD of ACIII on the periplasmic side of the membrane (Extended Data Fig. 9). The structural model reveals a $\pi$-cation interaction between Trp188 of subunit III and Arg868 of ActB (Fig. 4b), both of which are conserved among organisms containing subunit III with a long loop between TM5 and TM6 (Extended Data Fig. 9). This specific and strong interaction stabilizes the ACIII-cyt $a_{3} a_{3}$ supercomplex and appears to be a second adaptation that enables supercomplex formation with ACIII. The contact between the periplasmic loop of subunit III of cyt $a a_{3}$ and ACIII is the only observed direct contact between the two complexes. The five well-resolved transmembrane a-helices of subunit III of cyt aas are angled away from ACIII with only the tip of TM6 of subunit III touching ActF, forming a wedge-like space between the membrane domains of ACIII and cyt $a a_{3}$. The fall-off of resolution in the portions of cyt $a a_{3}$ that are distant from the interface with ACIII suggests that there may be multiple conformations of the supercomplex that are all tethered by the loop in cyt $a a_{3}$. The loop could, therefore, serve as a hinge, allowing the membrane domains of ACIII and cyt $a_{3}$ to swing into contact transiently.

Using the location of TM3 to TM7 of subunit III within the supercomplex as a guide allows a model of the entire cyt $a_{3}$ to be placed within the density map for the supercomplex (Extended Data Fig. 9). In the resulting model, there is a considerable distance (56 $⿱$ ) between the heme $c$ in $\mathrm{ActE}$ and $\mathrm{Cu}_{\mathrm{A}}$ within subunit II of cyt aas. Electron transfer within the supercomplex does not require the addition of exogenous cyt $c$, which is also the case for the $b c c$-cyt $a_{3}{ }_{3}$ supercomplex from Corynebacterium glutamicum ${ }^{23}$. It is possible, though it seems unlikely, that there is a subset of conformations in which ActE comes close enough to cyt $a a_{3}$ for direct electron transfer. It is noteworthy that the monoheme domain of ActA has substantial sequence homology ( $\sim 30 \%$ identity) with the heme $c$ domain that is present at the $\mathrm{C}$ terminus of subunit II of cyt caa 3 from Thermus thermophilus. This observation suggests that the ActA monoheme domain, which we postulate to be highly mobile in the 
structure (see above), may be able to interact with subunit II of cyt $a a_{3}$ and shuttle electrons from the ActE monoheme domain to subunit II of cyt $a_{3}$. Hence, electron transfer within the supercomplex may require the monoheme domain of ACIII to swing back and forth between ACIII and cyt $a a_{3}$ to shuttle electrons (Fig. 4c). Additional experimental work will be required to test this model and, indeed, to determine the physiological advantage of forming the supercomplex.

\section{METHODS}

\section{Bacterial strain and growth conditions}

Flavobacterium johnsoniae ATCC $17061^{\mathrm{TM}}$ strain UW101 was used in this study. The strain was a kind gift from Dr. Mark McBride at the University of Wisconsin, Milwaukee. The cells were grown in casitone-yeast extract (CYE) medium at $30{ }^{\circ} \mathrm{C}$ under high aerobic conditions (500 ml cultures in $2 \mathrm{~L}$ flasks) ${ }^{24}$.

\section{Membrane preparation and protein purification}

Cells grown overnight were collected by centrifugation $(14,000 \times \mathrm{g}$ for $10 \mathrm{~min})$. The cell pellet from $12 \mathrm{~L}$ of culture $(\sim 2.5 \mathrm{~g} / \mathrm{L})$ was resuspended in $\sim 200 \mathrm{ml}$ of $20 \mathrm{mM}$ Tris-HCl buffer, pH 8 (buffer A) with $5 \mathrm{mM} \mathrm{MgSO}_{4}$, DNAse I (Sigma) and a protease inhibitor cocktail (Sigma). This suspension was passed three times through a Microfluidizer at a pressure of $80,000 \mathrm{psi}$ to disrupt the cells. The cell extract was centrifuged at $14,000 \times \mathrm{g}$ for $10 \mathrm{~min}$ to remove unbroken cells. Membranes were obtained after centrifugation at 185,500 $\times \mathrm{g}$ for $4 \mathrm{~h}$. Under the above growth conditions, the membranes contained ACIII, cyt $a_{3} a_{3}$ and cyt $b d$. The membrane pellet was solubilized by using either a traditional detergent or the SMA copolymer.

\section{Purification using Triton X-100 and DDM}

The membrane pellet was resuspended in buffer $\mathrm{A}(\sim 50 \mathrm{mg} / \mathrm{ml})$ along with $300 \mathrm{mM} \mathrm{NaCl}$, and solubilized by the addition of Triton X-100 (Fisher Scientific) to a final concentration of $4 \%$. The solution was incubated at $4{ }^{\circ} \mathrm{C}$ for $2 \mathrm{~h}$ with mild agitation. The suspension was cleared by centrifugation at $185,500 \times \mathrm{g}$ for $1 \mathrm{~h}$, after which the detergent was diluted 4 -fold by adding three volumes of buffer A to the supernatant. The diluted supernatant was then added to a chromatography column containing $10 \mathrm{ml}$ of Ni-NTA resin (Qiagen) preequilibrated with $20 \mathrm{mM}$ Tris- $\mathrm{HCl} \mathrm{pH} 8$ containing $0.05 \%$ Triton X-100 and $0.15 \mathrm{M} \mathrm{NaCl}$ (buffer B). The resin was washed with about 10 column volumes of buffer B to remove any unbound sample. Detergent exchange to DDM (Anatrace) was carried out by washing with buffer B containing $0.05 \%$ DDM instead of Triton X-100 (buffer C). The column was further washed with 5 column volumes of buffer $\mathrm{C}$ containing $10 \mathrm{mM}$ imidazole to remove the loosely bound proteins from the resin. The proteins that were well bound to the resin were eluted using $100 \mathrm{mM}$ imidazole in buffer $\mathrm{C}$. The eluent was concentrated to $\sim 3 \mathrm{ml}$ using Amicon Ultra-15 filters (Millipore) with a $100 \mathrm{kDa}$ cutoff. The excess imidazole was removed by dialysis against buffer $\mathrm{C}$. The yield of protein obtained was about $0.3 \mathrm{mg} / \mathrm{L}$ of ACIII and $0.16 \mathrm{mg} / \mathrm{L}$ of cyt $a_{3}$ from $12 \mathrm{~L}$ of culture. When indicated, the proteins were further purified by gel filtration chromatography using Superdex 200 10/300 GL (GE 
Healthcare Life Sciences). The purified proteins were stored at $-80^{\circ} \mathrm{C}$ after adding glycerol to a final concentration of $10 \%$.

\section{Purification using SMA copolymer}

The SMA copolymer SMA ${ }^{\circledR}$ 3000HNA (styrene maleic acid copolymer ca. 3:1 molar ratio of styrene:maleic acid) was kindly provided as a gift from Dr. Terry Bricker (Louisiana State University, Baton Rouge, LA) who used SMA copolymer made by Cray Valley USA LLC (now Total Petrochemicals \& Refining USA Inc.) successfully for the studies of photosystem from spinach thylakoids ${ }^{25}$. Additional SMA ${ }^{\circledR} 3000 \mathrm{HNA}$ was kindly provided by Total Petrochemicals \& Refining USA (Houston, TX) as an aqueous solution of $25.6 \%$ (w/v) SMA. We also used a similar product, Xiran SL25010 S25, kindly provided by Polyscope Polymers B. V., with similar results. These polymer preparations are provided as aqueous solutions of the sodium salt, and the polymer solutions were simply diluted to the final desired percentage to use directly for solubilization of membranes. The purification protocol with the SMA copolymer was similar to that described with detergents with the following differences. After the membrane pellet was resuspended, the SMA solution was added dropwise to a final concentration of $1 \%$ with continuous stirring. After incubation for $1 \mathrm{~h}$ at room temperature, the solution was centrifuged at $185,500 \times \mathrm{g}$ for $1 \mathrm{~h}$ to remove unsolubilized particles. The supernatant was loaded directly to the Ni-NTA column equilibrated with $20 \mathrm{mM}$ Tris- $\mathrm{HCl} \mathrm{pH} 8,0.15 \mathrm{M} \mathrm{NaCl}$. The remaining steps of the purification were as described above. Following solubilization of the membrane suspension with $1 \%$ SMA $3000 \mathrm{HNA}$, no additional SMA or detergents were added and were not needed to maintain the solubilized proteins in solution. The yield of protein after the use of the SMA copolymer for solubilization was about $0.5 \mathrm{mg} / \mathrm{L}$ for ACIII and about $0.15 \mathrm{mg} / \mathrm{L}$ for cyt $\mathrm{aa}_{3}$ from $12 \mathrm{~L}$ of culture.

\section{Analytical methods}

The total protein concentration was determined using the BCA kit (Thermo Scientific, Pierce Protein Research Products). The UV-visible spectra of the oxidized and reduced proteins were recorded on an Agilent Technologies spectrophotometer (model 8453). The pyridine hemochrome assay ${ }^{26}$ was used to determine the concentration of hemes present in the protein samples. The total heme $c$ concentration was divided by seven to calculate the ACIII concentration and the total heme a concentration was divided by two to calculate the cyt $a a_{3}$ concentration. The purified protein was analyzed by SDS-PAGE using 4-20\% precast gels (Nusep Tech). Heme staining was carried out using 3,3',5,5' -tetramethyl benzidene (TMBZ) ${ }^{27}$. The supercomplex was visualized by blue native PAGE (BN-PAGE) using a $4-$ $16 \%$ gel (Novex, Life Technologies) with Bis-Tris buffer. The entire gel was stained with Coomassie blue, and then fixed with $30 \%$ methanol and $10 \%$ glacial acetic acid. The gel was destained with $8 \%$ glacial acetic acid to visualize the bands. Peptide mass spectrometry and analyses was carried out by Dr. Peter Yau at the Roy J. Carver Biotechnology Center (CBC) at the University of Illinois at Urbana-Champaign.

\section{Oxygen consumption assay}

Oxygen consumption was measured using a Clark electrode (Strathkelvin) in a $1 \mathrm{ml}$ chamber at $25^{\circ} \mathrm{C}$ as described in ${ }^{28}$. The reaction mix consisted of $100 \mu \mathrm{M}$ ubiquinone-1 
$\left(\mathrm{Q}_{1}\right)$ (Sigma-Aldrich) and $5 \mathrm{mM}$ dithiothreitol (DTT) in air-saturated 0.1 $\mathrm{M}$ potassium phosphate buffer, $\mathrm{pH} 7.5$ with $150 \mathrm{mM} \mathrm{NaCl}$. The reaction was started by adding the purified protein into the chamber. The initial concentration of oxygen was calculated to be $237 \mu \mathrm{M}$.

\section{Quinol:cytochrome c oxidoreductase activity}

The quinol:cytochrome $c$ oxidoreductase activity of the ACIII was measured spectrophotometrically as described in ${ }^{29}$. The reaction was carried out in a $2 \mathrm{ml}$ anaerobic cuvette, at $25^{\circ} \mathrm{C}$ in $50 \mathrm{mM}$ potassium phosphate buffer, $\mathrm{pH} 7.5$ in the presence of $50 \mu \mathrm{M}$ horse heart cytochrome $c$ (Sigma-Aldrich) and $200 \mu \mathrm{M} \mathrm{KCN}$. Ubiquinol- $1\left(\mathrm{Q}_{1} \mathrm{H}_{2}\right)$ or reduced vitamin $\mathrm{K}_{2}$ (Sigma-Aldrich) were used as quinol substrates and, in each case, the quinone was reduced using sodium borohydride according to the method by Ragan ${ }^{30}$. The reaction was started by the addition of $100 \mu \mathrm{M}$ of reduced quinone.

\section{EPR spectroscopy}

The purified ACIII-cyt $a a_{3}$ supercomplex was extensively dialyzed against $20 \mathrm{mM}$ Tris- $\mathrm{HCl}$ buffer, $\mathrm{pH} 8$, with $150 \mathrm{mM} \mathrm{NaCl}$ and $1 \mathrm{mM}$ EDTA to eliminate adventitious transition metal ions. The sample was concentrated in an Amicon filter to $150 \mu \mathrm{l}$ with a final ACIII concentration of $\sim 60 \mu \mathrm{M}$. The air-oxidized sample was directly transferred to an X-band EPR tube and subsequently frozen in liquid nitrogen. The sample was oxidized completely by the addition of $2 \mathrm{mM}$ potassium ferricyanide. Glycerol (5\%) was present in all EPR samples. Continuous Wave (CW) EPR measurements were carried out on an X-band Varian EPR-E122 spectrometer at the Electron Paramagnetic Resonance facility at the University of Illinois at Urban-Champaign. Cryogenic conditions below $77 \mathrm{~K}$ were achieved with a Lakeshore 331 temperature controller using a regulated flow of helium gas.

\section{Metal Analysis}

Metal Analysis was carried out using inductively coupled plasma mass spectrometry (ICPMS) as previously described ${ }^{31,32}$.

\section{Optical Redox Titration}

Full spectrum UV-visible redox titrations were performed to determine the midpoint potentials $\left(E_{m}^{o}\right.$ ) of the redox-active cytochromes in the DDM-solubilized ACIII-cyt aa 3 supercomplex ${ }^{33,34}$. The purified supercomplex was suspended in $4 \mathrm{ml}$ of $50 \mathrm{mM}$ potassium phosphate buffer ( $\mathrm{pH}$ 7.0) to a concentration of $3 \mu \mathrm{M}$ with $25 \mu \mathrm{M}$ each of following redox mediators; benzyl viologen $\left(E_{m, 7}=-350 \mathrm{mV}\right)$, anthraquinone-2-sulfonate $\left(E_{m, 7}=-225\right.$ $\mathrm{mV})$, 2-hydroxy-1,4-naphthoquinone $\left(E_{m, 7}=-220 \mathrm{mV}\right), 9,10$-anthroquinone-2,6disulfonate $\left(E_{m, 7}=-185 \mathrm{mV}\right)$, duroquinone $\left(E_{m, 7}=5 \mathrm{mV}\right), N$-ethylphenazonium ethosulfate $\left(E_{m, 7}=65 \mathrm{mV}\right), N$-methylphenazonium methosulfate $\left(E_{m, 7}=85 \mathrm{mV}\right)$, diaminodurene $\left(E_{m, 7}=275 \mathrm{mV}\right), 2,6$-dimethyl benzoquinone $\left(E_{m, 7}=180 \mathrm{mV}\right), 1,2$ napthoquinone $\left(E_{m, 7}=143 \mathrm{mV}\right), 1,4$-napthoquinone $\left(E_{m, 7}=36 \mathrm{mV}\right)$ and potassium ferricyanide $\left(E_{m, 7}=435 \mathrm{mV}\right){ }^{35}$. Titrations were performed with an anaerobic stirred cuvette and the solution potential was adjusted by injecting aliquots of $10 \mathrm{mM}$ sodium dithionite or potassium ferricyanide as reductant and oxidant, respectively. Spectra were taken at 
approximately $10-20 \mathrm{mV}$ increments over the titration range indicated. Spectroscopic changes of the a-bands of the hemes upon reduction or oxidation were monitored at the peak maxima to determine the midpoint potentials of each class of heme center. The data sets were analyzed using Origin ${ }^{\mathrm{TM}}$ (Origin Lab Corporation) to determine spectral components and fit titration curves using the Nernst equation ${ }^{35}$.

\section{EM sample preparation}

Holey carbon film-coated EM grids were nanofabricated with regular arrays of 500 to 800 $\mathrm{nm}$ holes ${ }^{36}$ and coated with an additional layer of gold. Cryo-EM specimens were prepared with a FEI Vitrobot grid preparation robot at $4{ }^{\circ} \mathrm{C}$ and $100 \%$ humidity by applying $3 \mu \mathrm{l}$ of sample ( $3 \mathrm{mg} / \mathrm{ml})$ to glow-discharged grids, allowing the grids to equilibrate for $1 \mathrm{~s}$, and blotting for $12 \mathrm{~s}$ before freezing in a liquid ethane/propane mixture $(1: 1 \mathrm{v} / \mathrm{v})^{37}$. Grids were subsequently stored in liquid nitrogen before shipping to the New York Structural Biology Center for imaging with a FEI Titan Krios electron microscope equipped with a Gatan K2 Summit camera and automated with Leginon ${ }^{38}$.

\section{EM data acquisition}

Movies were acquired in electron counting mode with a pixel size of $1.1 \AA$, an exposure rate of 7.4 electrons/pixel/s, and a total exposure time of $10 \mathrm{~s}$ divided in 40 frames (418 movies) or 50 frames (1599 movies). Frame alignment and exposure weighting were performed with Motioncor $2^{39}$. After screening averages from the aligned movies, 475 movies were discarded because of excessive movement, low defocus, high defocus, or over-focus. CTF parameters were estimated from the exposure-weighted averages of movie frames with CTFFIND $4^{40}$.

\section{Image processing}

3044 particle images were manually selected and subjected to 2D classification with Relion $1.4^{41}$. The resulting 2D classes were used as templates for automatic selection of 899405 particle images ${ }^{42}$. The number of particle images was reduced to 693416 by further 2D classification. Subsequent image processing was carried out in cryoSPARC $C^{43}$. An initial map of ACIII-cyt $a a_{3}$ was obtained by ab initio 3D classification, refined to $4.1 \AA$ resolution, and used as a reference for the multi-refine procedure in cryoSPARC producing initial maps of the ACIII and ACIII-cyt aa3 structures. 164239 particle images were used to refine the ACIII-cyt aa map to $3.4 \AA$ A resolution, but this map showed the cyt aa portion of the complex with lower-density than the ACIII part. Maps with uniform density for ACIII-cyt $a a_{3}$ and ACIII, both at $3.6 \AA$ resolution, were calculated from 81530 and 51547 particle images, respectively.

\section{Model building}

The $3.4 \AA$ resolution density map was used for the de novo model building of ACIII. The density map was first segmented with UCSF Chimera ${ }^{44}$ to facilitate the identification of subunits. The connectivity of each segmented map was further examined and the result was compared with topology predictions from topocons ${ }^{45}$ and secondary structure prediction from $J_{\text {pred }}{ }^{46}$ to validate the subunit assignment and identify the directionality of peptide 
chain. With this information, model building was carried out manually in $\mathrm{Coot}^{77}$. Individual chains were first traced in $\mathrm{Ca}$ baton mode. Readily interpretable features from the density map, including regions rich in bulky residues, triacylated cysteines, and axial ligands of heme $c$, were used to register the structure to the sequence. Stretches of $\sim 20$ amino acids were built progressively around these registration points and assembled as a single chain in Coot. All six subunits of ACIII were combined and refined with phenix.real_space_refine ${ }^{48}$. For cofactors, the starting models were taken from CCP4 ligand library directly. Cofactors were docked to the density map with Coot and merged with the apo protein structure. The complete structure was then refined with phenix.real_space_refine with geometric constraints for the protein-cofactor coordination. The final model was further examined in Coot to remove amino acid sidechains with ambiguous orientations and further validated with MolProbity ${ }^{49}$ and EMringer ${ }^{50}$. All identified lipids with two acyl tails were modeled as phosphatidylethanolamine (PE) with palmitoyl tail. The conformation of PE was refined with interactive Molecular Dynamics Flexible Fitting (iMDFF) in the presence of protein structure using $V M D^{51}$. Lipid tails were then truncated according to the density map.

The 3.6 A resolution ACIII-cyt $a a_{3}$ density map was used for the model building of cyt $a a_{3}$. Part of the subunit III loop region was manually built in Coot. Homology models for individual subunits were generated with the Raptor $X \operatorname{server}^{52}$ and docked into the density map with UCSF Chimera. The model for ACIII-cyt $a a_{3}$ supercomplex was assembled by fitting the ACIII structure to the ACIII-cyt $a a_{3}$ map and placing the cyt $a a_{3}$ structure from $R b$. sphaeroides (PDB 1M56) into the map based on the position of cyt aaz subunit III.

\section{Bioinformatic analysis}

Homologous protein sequences were retrieved using the NCBI blastp server ${ }^{53}$. The blastp results were analyzed in with pandas and biopython modules. Sequence hits were filtered based on coverage and sequence identity. Representative sequences were selected based on sequence identity to maintain the variations in sequence and aligned using the Clustal omega server $^{54}$. Figures for sequence alignment were prepared using the ESPript 3.0 server $^{55}$.

\section{Simulation system preparation}

The initial ACIII structure for the MD simulation was obtained from the refined structure determined by cryo-EM. Eleven 1-palmitoyl-2-oleoyl-sn-glycero-3-phosphoethanolamine (POPE) lipids resolved by cryo-EM were added to the ACIII system, which was subsequently embedded in a POPE membrane bilayer, solvated with the TIP3P water model $^{56}$, and ionized with $150 \mathrm{mM} \mathrm{NaCl}$.

\section{ReMDFF simulation}

Resolution-exchange Molecular Dynamics Flexible Fitting (ReMDFF) ${ }^{57}$ was used for structure refinement, with the CHARMM 36m force field for proteins ${ }^{58}$ and CHARMM 36 force field for lipids ${ }^{59}$. Force field parameters for hemes and iron sulfur cluster came from previous studies ${ }^{60-63}$. The fitting was performed in vacuum in the presence of a grid potential derived from the experimental density map (coupling factor 0.3 ). Secondary structure restraints, cis-peptide bond restraints, and chirality restraints were applied to the protein. Hemes and iron-sulfur clusters were harmonically restrained $\left(\mathrm{k}=50 \mathrm{kcal} / \mathrm{mol} / \AA^{2}\right)$. 
A Langevin thermostat ${ }^{64}$ was used for maintaining the average temperature at $80 \mathrm{~K}$. The MD integration time step was $1 \mathrm{fs}$. A cutoff radius for nonbonded interactions was set to 10 $\AA$ with a switching function taking effect at $9 \AA$. A total of six replicas were used together with six grid potentials of decreasing resolutions. Each was first energy-minimized for 2000 steps and then equilibrated for 1 ps. Finally, 2000 replica exchanges were attempted with 1 ps between attempts.

\section{MD simulation}

The ACIII systems were simulated with NAMD 2.12 using the same force field parameters as in ReMDFF. The system was energy-minimized for 3000 steps using the conjugated gradient algorithm ${ }^{65}$ with linear searching ${ }^{66}$, and equilibrated for $0.5 \mathrm{~ns}$ to relax lipid tail group atoms while keeping the lipid phosphorus atoms and protein (including hemes and iron-sulfur clusters) heavy atoms harmonically restrained $\left(\mathrm{k}=1 \mathrm{kcal} / \mathrm{mol} / \AA^{2}\right)$. This procedure was followed by a 10-ns simulation to allow lipids to relax around the proteins while keeping the protein backbone and heavy atoms from iron-sulfur clusters and hemes harmonically restrained $\left(\mathrm{k}=1 \mathrm{kcal} / \mathrm{mol} / \AA^{2}\right)$. Restraints were gradually released over the next $5 \mathrm{~ns}$ and the simulation continued without any biasing potential for a total of $250 \mathrm{~ns}$. The angles in the iron-sulfur clusters were harmonically restrained to their initial values $(\mathrm{k}=300$ $\mathrm{kcal} / \mathrm{mol} / \mathrm{deg}$ ) throughout the simulation.

\section{Data availability}

All relevant data are available from the corresponding authors upon reasonable request and/or included in the manuscript or Supplementary Information. Three cryo-EM maps mentioned in this work have been deposited in the Electron Microscopy Data Bank (EMDB) under accession codes EMD-7286 (Combined), EMD-7447 (ACIII-cyt $a a_{3}$ ), EMD-7448 (ACIII). The coordinates of the atomic model of the Alternative Complex III built from EMD-7286 have been deposited in the Protein Data Bank (PDB) under accession code 6BTM. 


\section{Extended Data}

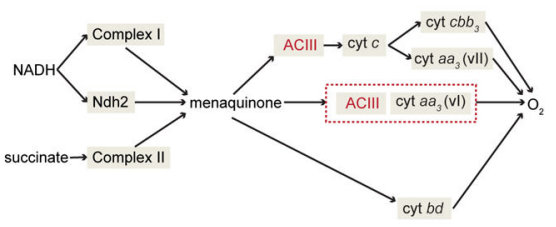

b

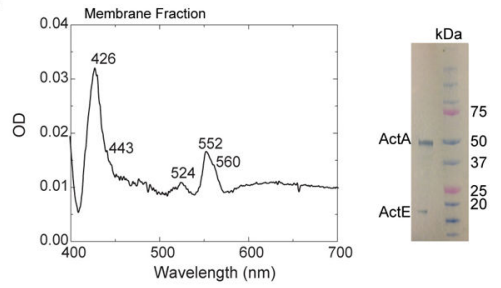

c

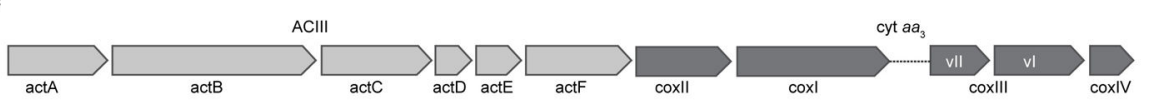

d

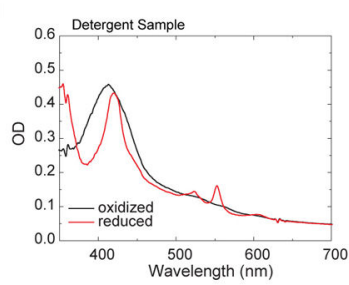

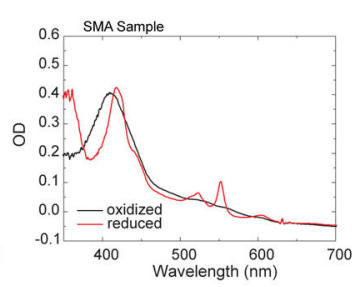

Extended Data Figure 1. Expression and spectroscopic characterization of the ACIII/cyt $a a_{3}$ supercomplex

a, A schematic representation of the respiratory chain of $F$. johnsoniae. b, UV/visible spectroscopy and SDS-PAGE with the membranes from $F$. johnsoniae. On the left is the difference spectrum of the membranes of $F$. johnsoniae was obtained from the spectrum of the air-oxidized membranes and the spectrum after reduction with dithionite. The wavelengths associated with the heme peaks are $605 \mathrm{~nm}, 560 \mathrm{~nm}, 552 \mathrm{~nm}$ and a broad peak at $630 \mathrm{~nm}$ for hemes $a, b, c$ and $d$ respectively. On the right is the SDS-PAGE with the membranes followed by staining the gel for hemes shows bands corresponding to the cytochrome subunits ActA $(48 \mathrm{kDa})$ and ActE $(20 \mathrm{kDa})$ of ACIII but nothing corresponding to the cytochrome subunit $(\sim 35 \mathrm{kDa})$ from the $c b b_{3}$ oxidase. $\mathbf{c}$, The gene arrangement for the ACIII and the cytochrome oxidase $a_{3}$ genes in the $F$. johnsoniae genome. The genes for the subunits I and II from cyt $a a_{3}$ oxidase are found immediately downstream of those for the act genes of the ACIII. Two different versions of subunit III are denoted as vI and vII. d, Reduced and oxidized UV/visible spectra of the supercomplex in detergent and SMA nanodiscs. The dithionite reduced form of the samples is represented in red and shows the peaks for heme $c$ at $524 \mathrm{~nm}$ and $552 \mathrm{~nm}$ and those for heme $a$ at $443 \mathrm{~nm}$ and $605 \mathrm{~nm}$. e, Pyridine hemochrome assay of the ACIII: $a_{3}$ supercomplex in SMA nanodiscs. Plotted is the reduced-minus-oxidized difference spectrum of the pyridine hemochromes of the sample. Peaks at $520 \mathrm{~nm}$ and $550 \mathrm{~nm}$ are associated with heme $c$ and peak at $590 \mathrm{~nm}$ is associated with heme $a$. Quantitation from the spectrum shows a ratio of 10.6:1 between heme $c$ and heme $a$, which translates into a 3:2 ratio between ACIII and aa 3 assuming 7 heme $c$ per ACIII and 2 heme a per $a a_{3}$. Data in $\mathbf{b}$ are representative of two independent experiments with similar results and data in $\mathbf{d , e}$ are representative of six independent experiments with similar results. 

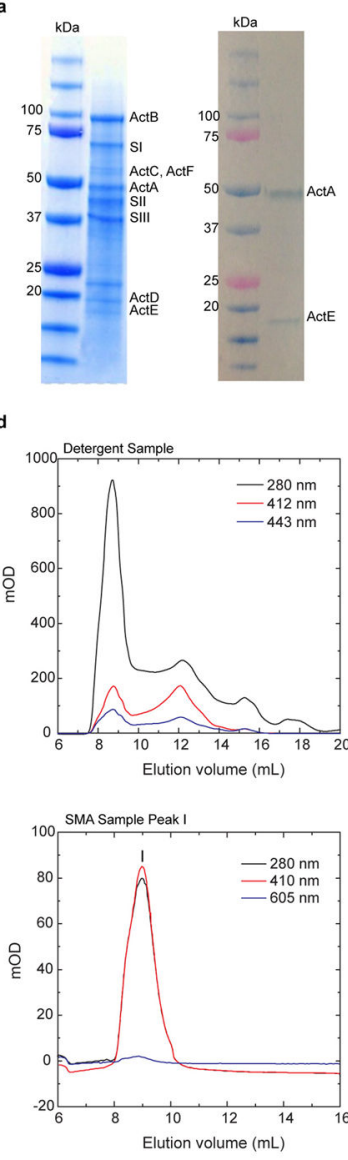
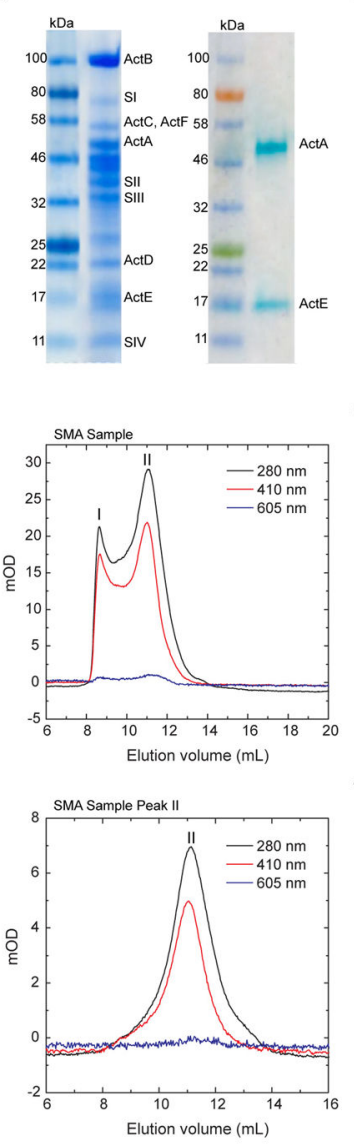
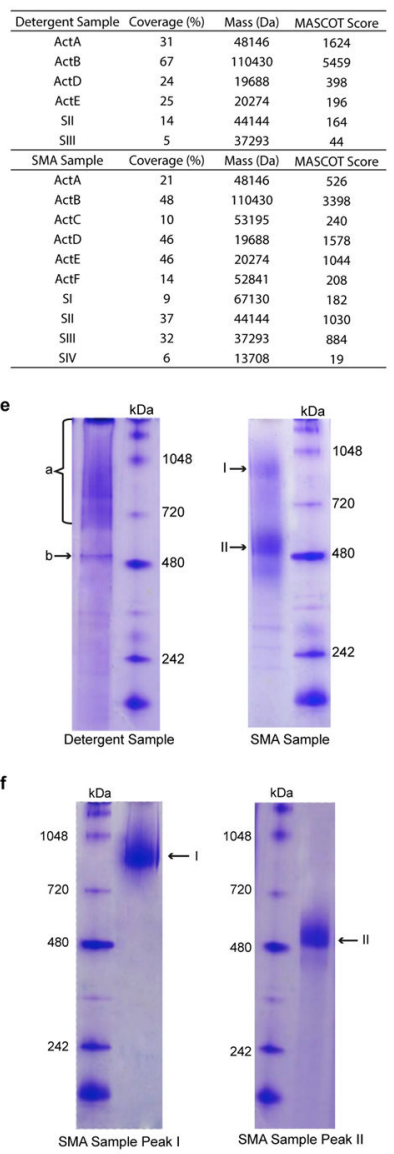

Extended Data Figure 2. Component and size analysis of the ACIII/cyt $\boldsymbol{a a}_{3}$ supercomplex a, SDS-PAGE of the detergent solubilized preparation followed by Coomassie staining (Left) and heme staining (Right). b, SDS-PAGE of the SMA nanodiscs preparation followed by Coomassie staining (Left) and heme staining (Right). c, Mass spectrometry results for the ACIII- $a_{3}$ supercomplex preparations. d, Size exclusion chromatography with the ACIII-aa 3 supercomplex from F.johnsoniae. (Top left) The detergent-solubilized sample showing traces for protein at $280 \mathrm{~nm}$, heme $c$ at $412 \mathrm{~nm}$ and heme $a$ at $443 \mathrm{~nm}$ respectively. (Top right) The sample isolated using the SMA copolymer showing traces for protein at $280 \mathrm{~nm}$, heme $c$ at $410 \mathrm{~nm}$ and heme $a$ at $605 \mathrm{~nm}$. I and II are the two peaks corresponding to two populations of the supercomplex. (Bottom left) The fraction containing peak I. (Bottom right) The fraction containing peak II. e, BN-PAGE with the ACIII: $a a_{3}$ supercomplex. Detergent solubilized ACIII-aa 3 supercomplex showing a band at around $500 \mathrm{kDa}$, a smear of possible aggregates and possibly ACIII by itself. The supercomplex in SMA nanodiscs shows two different populations. f, BN-PAGE with the two different populations of ACIII:aa 3 supercomplex in SMA nanodiscs purified from the size exclusion chromatography. The two chromatography peaks correspond to the two bands observed in the BN-PAGE. Data in a,b are representative of six independent experiments and those in $\mathbf{d , e , f}$ are representative of three independent experiments with similar results. 


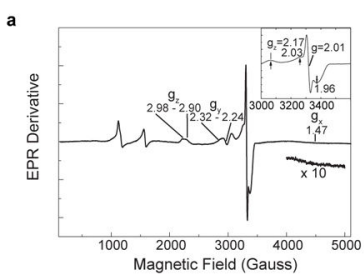

b
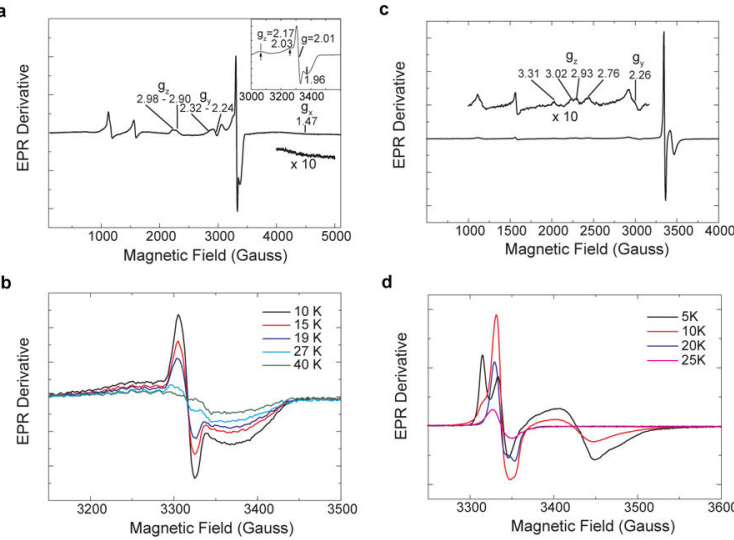

d
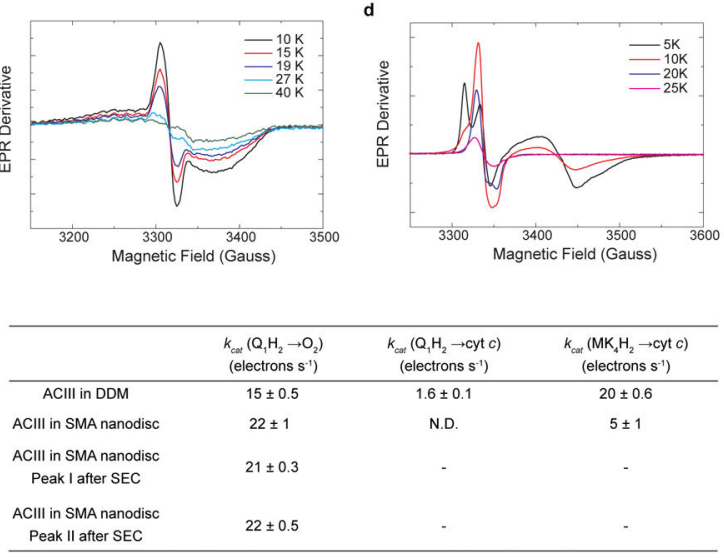
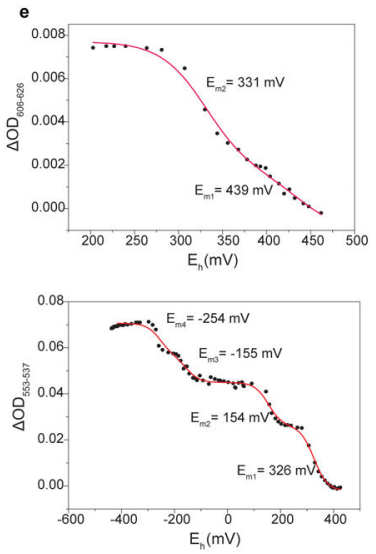

Extended Data Figure 3. Functional assays of the ACIII/cyt $a a_{3}$ supercomplex

a, The EPR spectrum of the air oxidized sample showing peaks of the $[3 \mathrm{Fe}-4 \mathrm{~S}]^{1+}$ cluster from $\mathrm{ACIII}$, the $\mathrm{Cu}_{\mathrm{A}}$ from the $\mathrm{aa}_{3}$ oxidase and low-spin hemes with overlapping $\mathrm{g}$ values. Insert is a zoomed view from $3000 \mathrm{G}$ to $3500 \mathrm{G}$ to better visualize the peaks from $\mathrm{Cu}_{\mathrm{A}}$ (black arrows) and $[3 \mathrm{Fe}-4 \mathrm{~S}]^{1+}$ cluster. The region between $4000 \mathrm{G}$ and $5000 \mathrm{G}$ is magnified 10 -times to show the broad $g_{x}$ trough of low-spin hemes. The measurement condition is 10 K, $9.267 \mathrm{GHz}, 2 \mathrm{~mW}$ microwave power and 20 Gauss modulation. b, The EPR spectra of the ferricyanide oxidized sample at various temperatures. The measurement condition is 9.257 GHz, $2 \mathrm{~mW}$ microwave power and 5 Gauss modulation. c, The EPR spectrum of the air oxidized sample showing peaks of iron sulfur clusters from ACIII and low spin hemes. The measurement condition is $10 \mathrm{~K}, 9.427 \mathrm{GHz}, 2 \mathrm{~mW}$ microwave power, 10 Gauss modulation. d, The EPR spectra of the air oxidized sample at various temperatures. The measurement condition is $9.427 \mathrm{GHz}, 2 \mathrm{~mW}$ microwave power, 5 Gauss modulation. e, Redox titration of the hemes in the ACIII and the aa 3 oxidase in supercomplex in DDM. The potentiometric titration of the $c$ hemes (Top) from the ACIII and the a hemes (Bottom) from the $a a_{3}$ oxidase. The $\mathrm{E}_{\mathrm{m}}$ values are indicated and the solid red line represents the Nernst fitting. $\mathbf{f}$, Steady state activity of preparations of the ACIII/cyt aa 3 preparations (The number of independent experiments, $n=6$ for ACIII in DDM and SMA nanodiscs and $n=3$ for Peak I and Peak II). Data are means \pm standard deviation (S.D.). Data in a-e are representative of three independent experiments with similar results. 

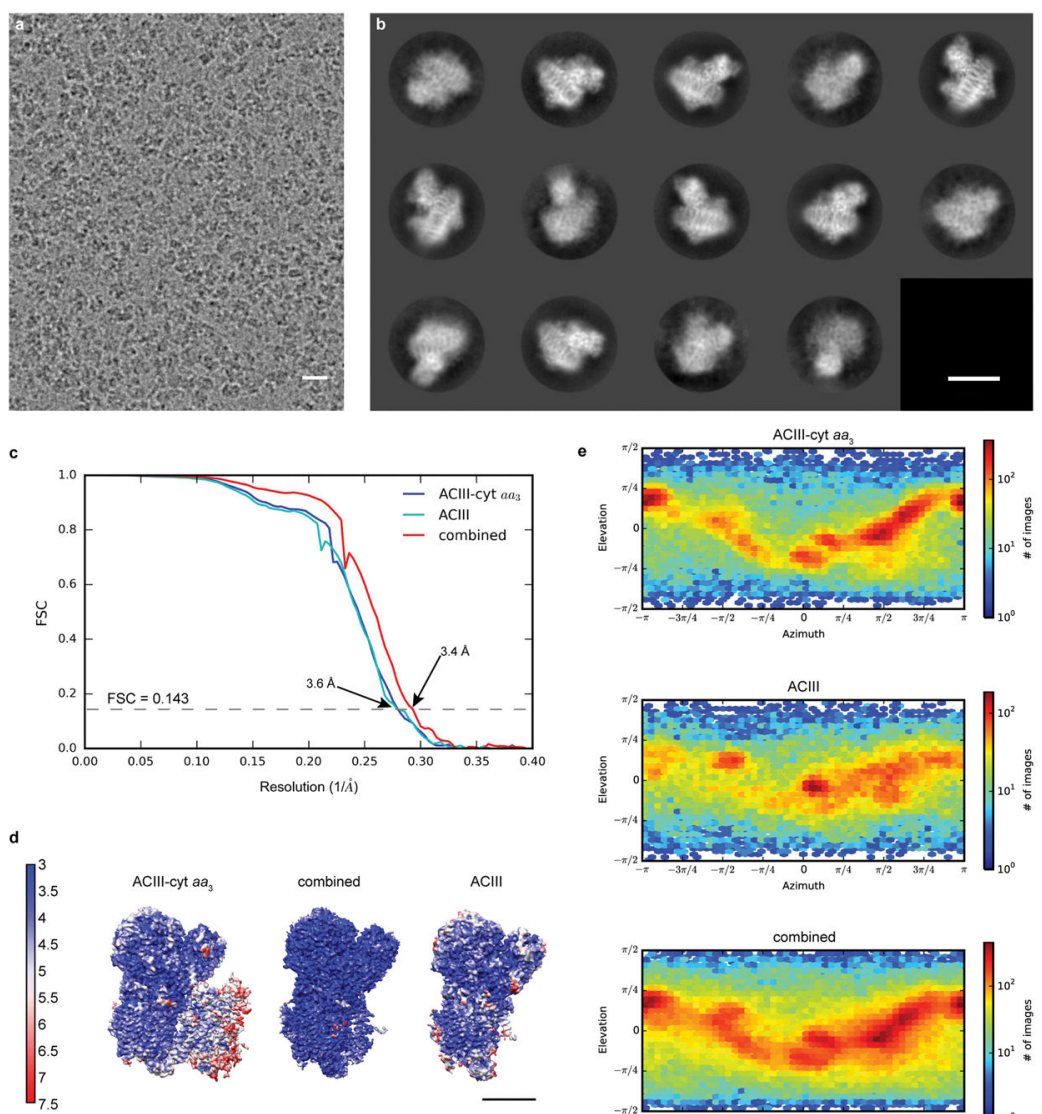
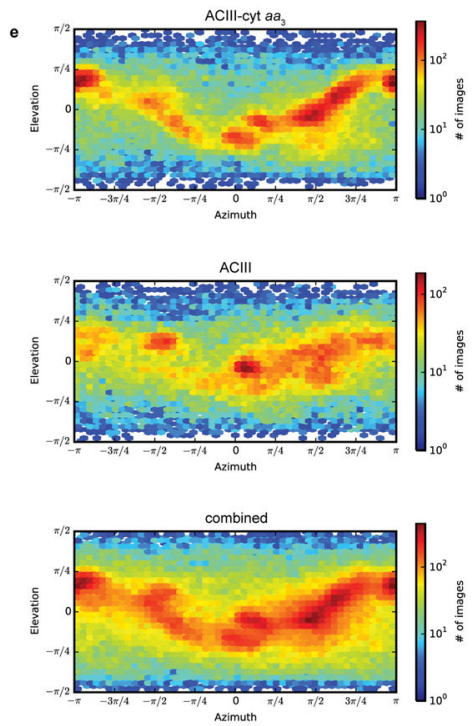

Extended Data Figure 4. single-particle cryo-EM of ACIII-cyt $a a_{3}$ supercomplex in SMA nanodiscs

a, Sum of an aligned movie of ACIII-cyt $a a_{3}$ supercomplex in an SMA nanodisc. The scale bar is $20 \mathrm{~nm}$. b. Two-dimensional class averages with the scale bar representing $10 \mathrm{~nm}$. c, Fourier shell coefficient (FSC) curves between two independently refined half-maps for the ACIII-cyt $a a_{3}$ map, ACIII map and combined map. d, Surface rendering maps colored according to local resolution, the scale bar is $5 \mathrm{~nm}$. e, Euler angle distributions of particles included in the calculation of the three final maps. Data collection and structure calculation were not repeated. 

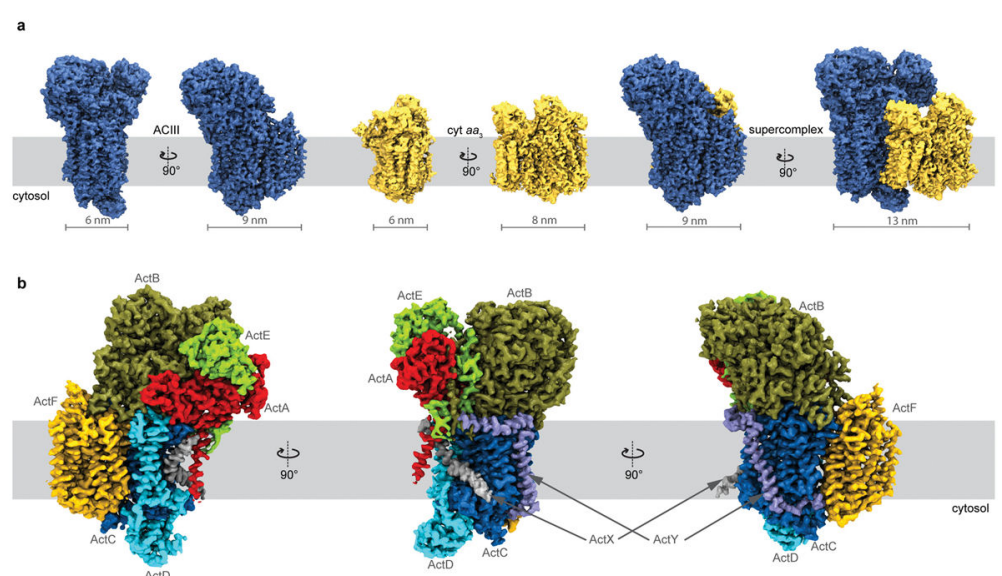

d

g

ActB/ActC
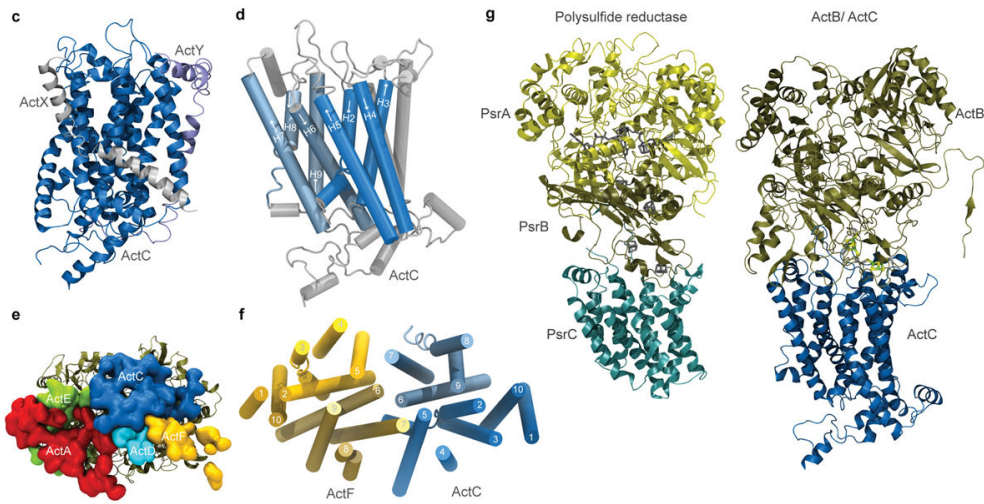

Extended Data Figure 5. Features observed in the cryo-EM density and the de novo structure of ACIII

a, Surface representations of ACIII, cyt $a a_{3}$ and the ACIII-cyt $a_{3}$ supercomplex. The density threshold is the same for ACIII and cyt $a a_{3}$. b. Different views of the ACIII density, colored by subunit. c, Two single-span transmembrane peptides of unknown origin and sequence, denoted ActX and ActY, are present in the structure in the vicinity of ActC. These have each been modeled as a polyalanine peptide. $\mathbf{d}$, $\mathbf{a}$-helices 2-10 of ActC form two four-helical upand-down bundles, colored in two different blue colors. a-helices 1 and 10 are colored gray and unlabeled. e, ActB, shown in cartoon, has contact with ActA, ActC, ActD, ActE and ActF. Surfaces are drawn from residues that are within $4 \AA$ of ActB and colored according to their chain. f, The transmembrane a-helices of ActC and ActF are arranged in a pseudo twofold rotation symmetry. g, Side-by-side comparison of the polysulfide reductase (PDB 2VPZ) and the assembly of ActB and ActC. These two structures are aligned based on PsrB, the domain containing four Fe-S clusters. 
a
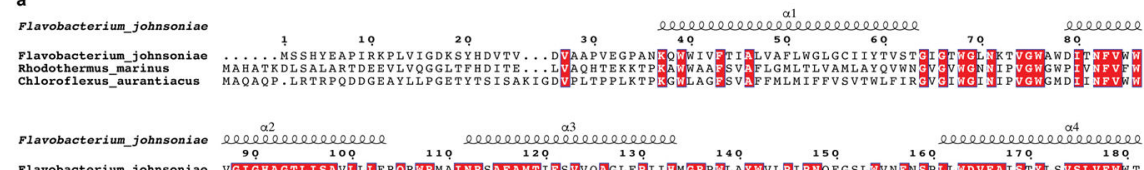

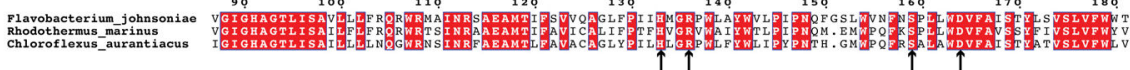

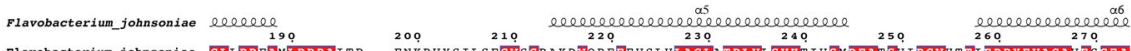

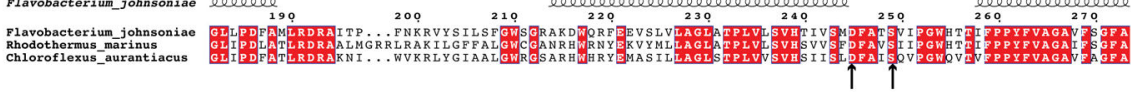

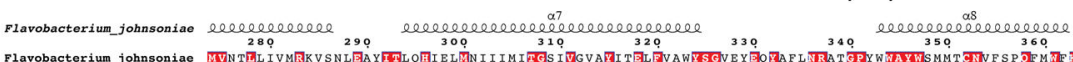

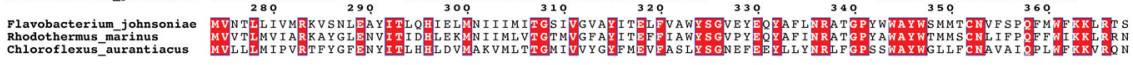

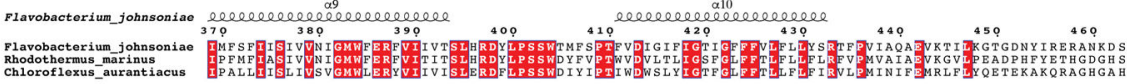

Flavobacterium_johnsoniac

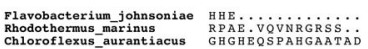

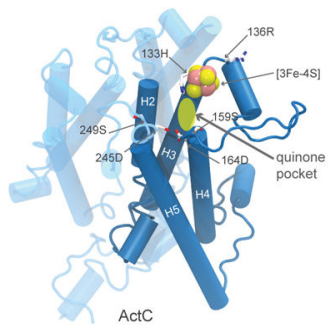

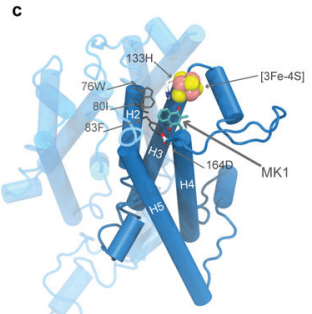

ActC

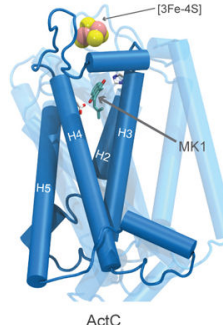

ActC

Extended Data Figure 6. quinone pocket in ActC

a, sequence alignment of the ActC from Flavobacterium johnsoniae, Rhodothermus marinus, and Chloroflexus aurantiacus. The transmembrane a-helices are labelled based on the structure of ACIII from Flavobacterium johnsoniae. The black arrows point to conserved polar residues that are within $15 \AA$ of the $[3 \mathrm{Fe}-4 \mathrm{~S}]$ cluster in ActB. b. Proposed quinone pocket based on the arrangement of conserved polar residues. c, Different views of the proposed quinone pocket with a docked menaquinone- 1 molecule. Hydrophobic residues near the menaquinone-1 (MK-1) head group are also shown. The crevice between $a$-helix 3 and a-helix 4 is a putative quinone entry pathway. 

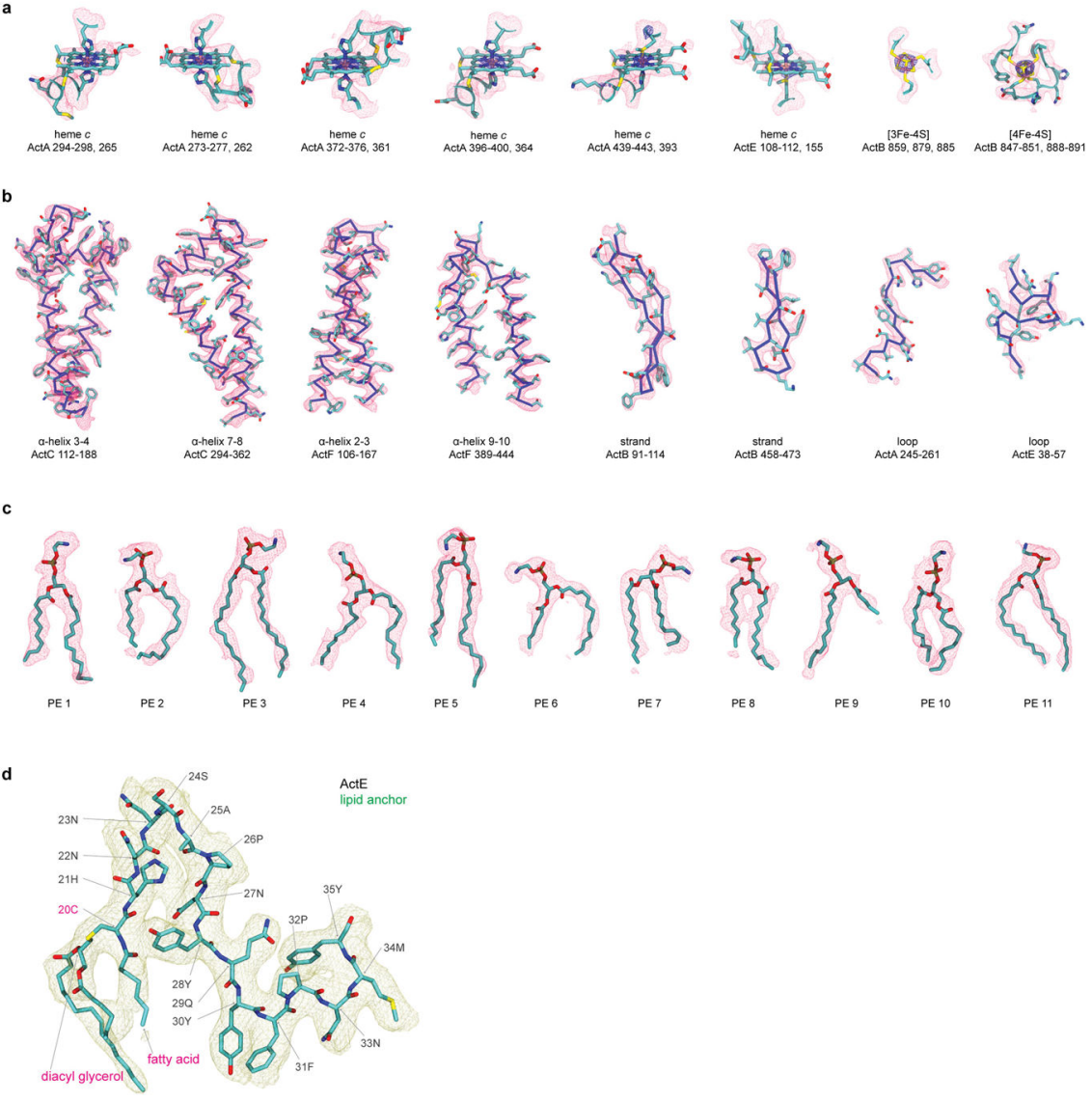

Extended Data Figure 7. Fitting of ACIII structure to cryo-EM density

a, Fitting of cofactors into the cryo-EM density. The blue mesh is drawn with a higher density threshold to reveal metal centers. The numberings of nearby amino acid residues, which are shown along with these cofactors, are listed below each cofactor. $\mathbf{b}$, fitting of different secondary structure elements to cryo-EM density. c, Eleven identified lipids are modelled as phosphatidylethanolamine molecules. $\mathbf{d}$, The triacylated cysteine at the $\mathrm{N}$ terminus of ActE shown along with 15 downstream amino acids. Notably, residue Tyr28 ${ }^{\mathrm{E}}$ is in contact with the covalent lipid of ActE. Attachment of ActE to the membrane may also be assisted by aromatic residues $\operatorname{Tyr} 30^{\mathrm{E}}$ and $\mathrm{Phe} 31^{\mathrm{E}}$, which appear to be inserted into the lipid bilayer. Throughout the MD simulation trajectory, these residues remain buried in the lipid bilayer. 

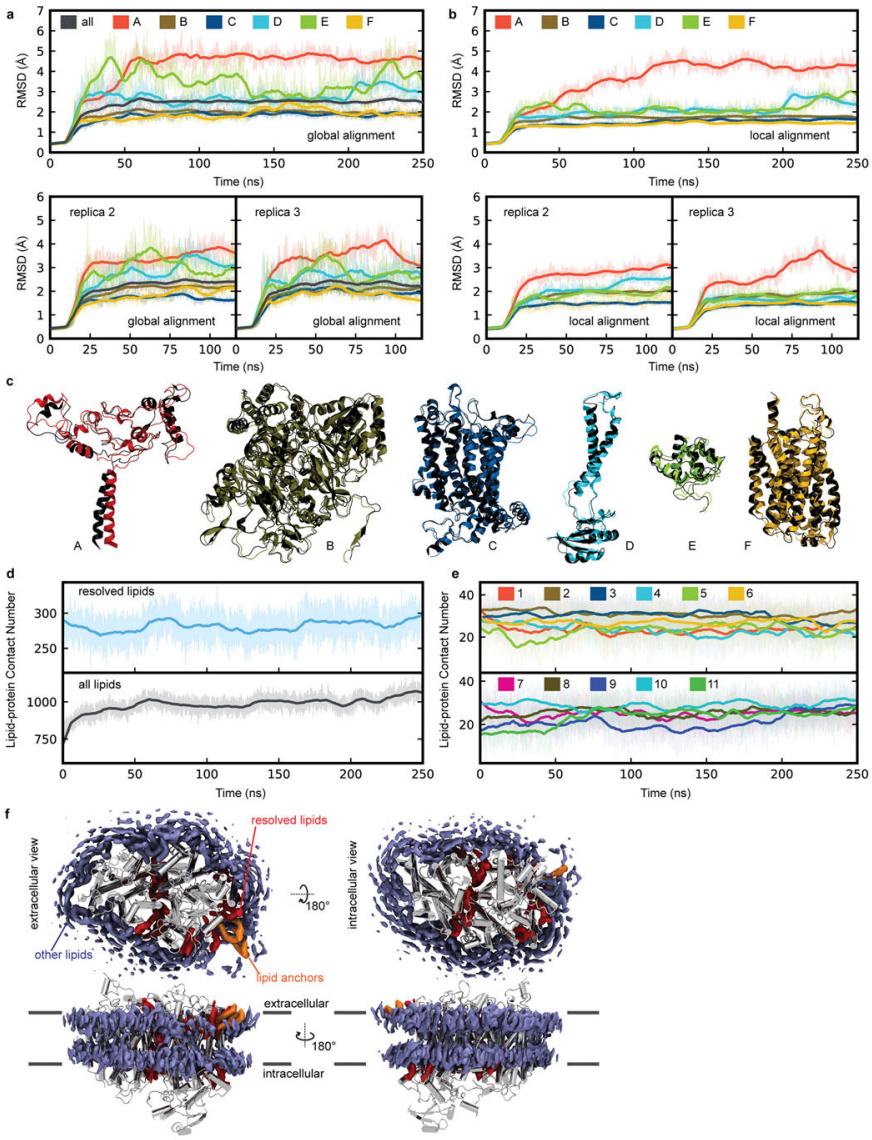

Extended Data Figure 8. Protein stability and lipid-protein interaction analysis based on molecular dynamics simulations

a, Root-mean-square deviation (RMSD) of the protein backbone heavy atoms for the entire ACIII complex and each subunit, aligned based on ACIII backbone heavy atoms from three independent molecular dynamics simulations. b, Same as $\mathbf{a}$, but aligned using the backbone heavy atoms of each subunits. c, Superposition of the initial (black) and final conformation (colored) of each subunit after $250 \mathrm{~ns}$ of simulation (aligned using backbone heavy atoms). d, The lipid-protein contact number defined by the number of lipid atoms within $4 \AA$ of the protein atoms calculated over the time course of the simulation. This contact number is either calculated for the eleven lipids resolved by cryo-EM (top) or all membrane lipids (bottom). e, The lipid-protein contact number for each of the eleven cryo-EM resolved lipids. f, Isosurfaces (50\%) of the atom occupancy map for the lipid anchors (orange), cryoEM resolved lipids (red), and other membrane lipids (purple), calculated using the last 230 ns simulation trajectory. The stronger the lipid-protein interactions, the longer the local residence time, which leads to higher atom-occupancy values. ACIII subunits $\mathrm{C}, \mathrm{D}$, and F are shown in silver. For all plots, the raw data are shown as translucent thin lines and the block-averages are shown as dark lines. 

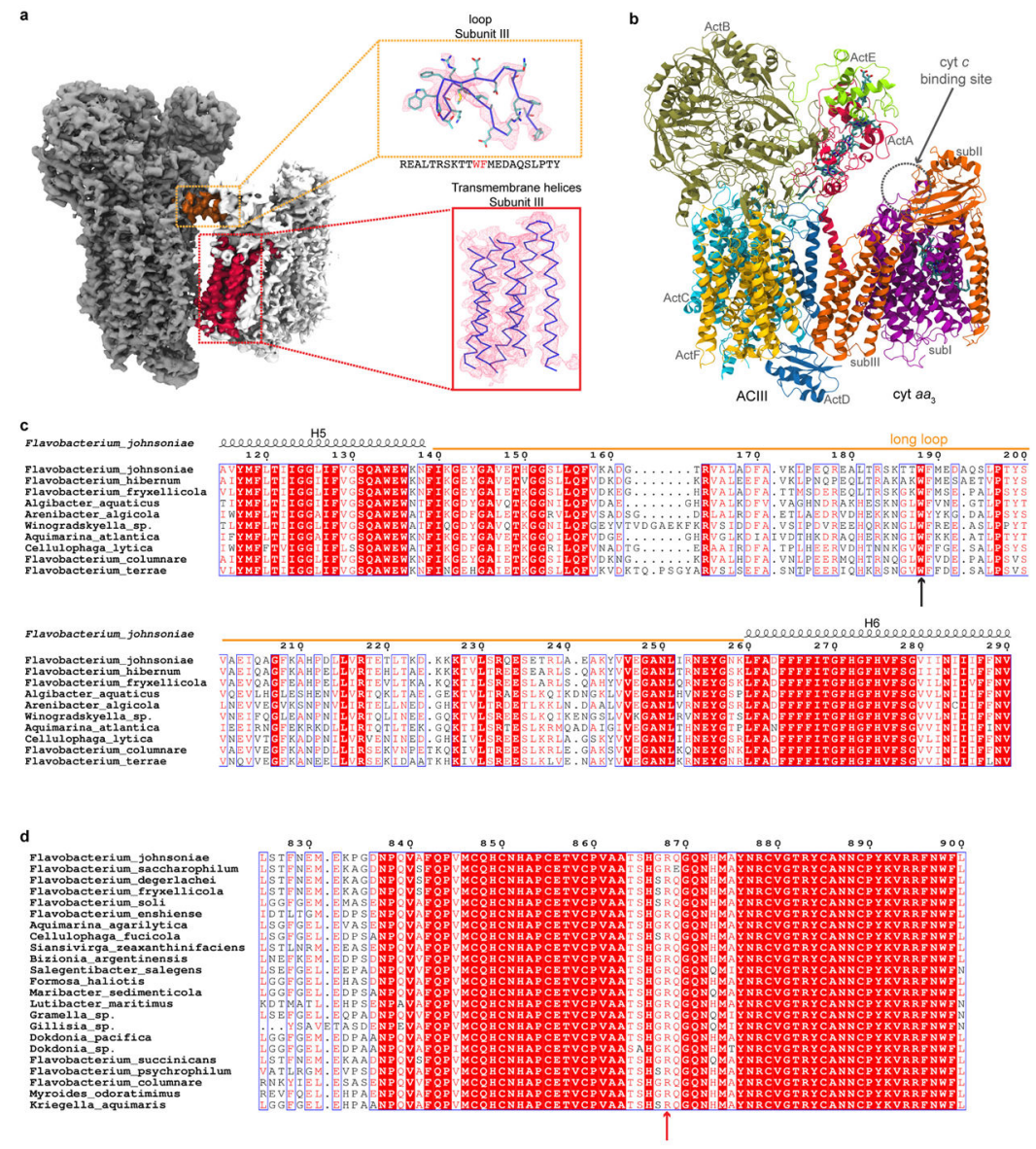

Extended Data Figure 9. Structural basis for the supercomplex formation between the ACIII and the cyt $a_{3}$

a, Two contact areas between the ACIII and the cyt aas: the transmembrane portion of subunit III (red) and the loop from subunit III (orange). A homology model of subunit III fits the transmembrane density. The loop is modeled to the cryo-EM density. The sequence of the peptide is also shown. Tryptophan 188 and phenylalanine 189 are used to register the density to the sequence. b, Model of the ACIII-cyt aa 3 supercomplex. The cyt $a a_{3}$ structure from $R b$. sphaeroides was positioned based on the transmembrane portion of subunit III. ahelices 1 and 2 of subunit III are missing in F.johnsoniae to avoid steric clashes with the ACIII structure. c, Sequence alignment of subunit III with a long loop (highlighted with the orange bar) between $a$-helix 5 and a-helix 6 (numbered according to subunit III from $R b$. sphaeroides). Tryptophan 188 (black arrow) is conserved. d, Sequence alignment of ActB from organisms with a long loop in their subunit III of cyt aa 3 oxidase. Arginine 868 (red arrow) is largely conserved with occasional substitution to lysine. 


\section{Extended Data Table 1}

Cryo-EM data collection, refinement and validation statistics

\begin{tabular}{|c|c|c|c|}
\hline & $\begin{array}{l}\text { Combined (EMDB-7286) } \\
\text { (PDB 6BTM) }\end{array}$ & ACIII-cyt aa3 (EMDB-7447) & ACIII (EMDB-7448) \\
\hline \multicolumn{4}{|l|}{ Data collection and processing } \\
\hline Magnification & $75,000 x$ & $75,000 \times$ & $75,000 \times$ \\
\hline Voltage $(\mathrm{kV})$ & 300 & 300 & 300 \\
\hline Electron exposure $\left(\mathrm{e}^{-} / \AA^{2}\right)$ & 61 & 61 & 61 \\
\hline Defocus range $(\mu \mathrm{m})$ & $0.8-5.0$ & $0.8-5.0$ & $0.8-5.0$ \\
\hline Pixel size $(\AA)$ & 1.1 & 1.1 & 1.1 \\
\hline Symmetry imposed & $\mathrm{C} 1$ & $\mathrm{C} 1$ & $\mathrm{C} 1$ \\
\hline Initial particle images (no.) & 899,405 & 899,405 & 899,405 \\
\hline Final particle images (no.) & 164,239 & 81,530 & 51,547 \\
\hline Map resolution $(\AA)$ & $3.4^{*}$ & $3.6^{*}$ & $3.6^{*}$ \\
\hline FSC threshold & 0.143 & 0.143 & 0.143 \\
\hline Map resolution range $(\AA)$ & $3.0-4.5$ & $3.0-6.0$ & $3.0-5.0$ \\
\hline \multicolumn{4}{|l|}{ Refinement } \\
\hline Initial model used (PDB code) & N/A & N/A & N/A \\
\hline Model resolution $(\AA)$ & $3.7^{\dagger}$ & N/A & N/A \\
\hline FSC threshold & 0.5 & & \\
\hline Model resolution range $(\AA)$ & 3.7 & N/A & N/A \\
\hline Map sharpening $B$ factor $\left(\AA^{2}\right)$ & -150.1 & -132.4 & -129.1 \\
\hline \multicolumn{4}{|l|}{ Model composition } \\
\hline Non-hydrogen atoms & 18,935 & & \\
\hline Protein residues & 2,361 & & \\
\hline Ligands & 10 & & \\
\hline \multicolumn{4}{|l|}{$B$ factors $\left(\AA^{2}\right)$} \\
\hline Protein & $126.4^{*}$ & & \\
\hline Ligand & $119.7^{*}$ & & \\
\hline \multicolumn{4}{|l|}{ R.M.S. deviations } \\
\hline Bond lengths ( $(\AA)$ & 0.009 & & \\
\hline Bond angles $\left({ }^{\circ}\right)$ & 1.26 & & \\
\hline \multicolumn{4}{|l|}{ Validation } \\
\hline MolProbity score & 1.36 & & \\
\hline Clashscore & 1.58 & & \\
\hline Poor rotamers $(\%)$ & 0.15 & & \\
\hline \multicolumn{4}{|l|}{ Ramachandran plot } \\
\hline Favored $(\%)$ & 92.7 & & \\
\hline Allowed (\%) & 7.3 & & \\
\hline Disallowed (\%) & 0.0 & & \\
\hline
\end{tabular}

Nature. Author manuscript; available in PMC 2018 October 25. 


\section{Supplementary Material}

Refer to Web version on PubMed Central for supplementary material.

\section{Acknowledgments}

This work was supported by funds from the National Institutes of Health (R01-HL016101 to R.B.G., P41GM104601, U54-GM087519, and R01-GM123455 to E.T.) and Canadian Institutes of Health Research (MOP-81294 to J.L.R.); J.L.R. was supported by the Canada Research Chairs program. Some of this work was performed at the Simons Electron Microscopy Center and National Resource for Automated Molecular Microscopy, supported by grants from the Simons Foundation (349247), and the National Institute of General Medical Sciences (P41-GM103310, S10-OD019994). Molecular dynamics simulations were performed at Blue Waters (ACI-1713784 to E.T.) and XSEDE (TG-MCA06N060 to E.T.). Blue Waters is supported by the National Science Foundation (OCI-0725070 and ACI-1238993) and the state of Illinois. XSEDE is supported by the National Science Foundation (ACI-1548562). We thank Mark Mcbride for kindly providing us with the Flavobacterium johnsoniae UW101 strain. We thank Bridget Carragher and Clint Potter for facilitating access to the Titan Krios and Zhening Zhang for collecting data. We also thank Mohammad Mazhab-Jafari for advice on building atomic models.

\section{References}

1. Pereira MM, Carita JN, Teixeira M. Membrane-bound electron transfer chain of the thermohalophilic bacterium Rhodothermus marinus: a novel multihemic cytochrome $b c$, a new complex III. Biochemistry. 1999; 38:1268-1275. [PubMed: 9930987]

2. Yanyushin MF, del Rosario MC, Brune DC, Blankenship RE. New class of bacterial membrane oxidoreductases. Biochemistry. 2005; 44:10037-10045. [PubMed: 16042380]

3. Refojo PN, Sousa FL, Teixeira M, Pereira MM. The Alternative Complex III: a different architecture using known building modules. Biochim Biophys Acta. 2010; 1797:1869-1876. [PubMed: 20416271]

4. Pereira MM, Refojo PN, Hreggvidsson GO, Hjorleifsdottir S, Teixeira M. The Alternative Complex III from Rhodothermus marinus - a prototype of a new family of quinol:electron acceptor oxidoreductases. FEBS Lett. 2007; 581:4831-4835. [PubMed: 17888426]

5. Gao X, Xin Y, Bell PD, Wen J, Blankenship RE. Structural analysis of Alternative Complex III in the photosynthetic electron transfer chain of Chloroflexus aurantiacus. Biochemistry. 2010; 49:6670-6679. [PubMed: 20614874]

6. Refojo PN, Ribeiro MA, Calisto F, Teixeira M, Pereira MM. Structural composition of Alternative Complex III: variations on the same theme. Biochim Biophys Acta. 2013; 1827:1378-1382. [PubMed: 23313414]

7. Xia D, et al. Crystal structure of the cytochrome $b c_{1}$ complex from bovine heart mitochondria. Science. 1997:277.

8. Rothery RA, Workun GJ, Weiner JH. The prokaryotic complex iron-sulfur molybdoenzyme family. Biochim Biophys Acta. 2008; 1778:1897-1929. [PubMed: 17964535]

9. Dietrich W, Klimmek O. The function of methyl-menaquinone-6 and polysulfide reductase membrane anchor (PsrC) in polysulfide respiration of Wolinella succinogenes. Eur J Biochem. 2002; 269:1086-1095. [PubMed: 11856339]

10. Hedderich R, et al. Anaerobic respiration with elemental sulfur and with disulfides. FEMS Microbiol Rev. 1998; 22:353-381.

11. Dorr JM, et al. Detergent-free isolation, characterization, and functional reconstitution of a tetrameric $\mathrm{K}^{+}$channel: the power of native nanodiscs. Proc Natl Acad Sci USA. 2014; 111:1860718612. [PubMed: 25512535]

12. Postis V, et al. The use of SMALPs as a novel membrane protein scaffold for structure study by negative stain electron microscopy. Biochim Biophys Acta. 2015; 1848:496-501. [PubMed: 25450810]

13. Dorr JM, et al. The styrene-maleic acid copolymer: a versatile tool in membrane research. Eur Biophys J. 2016; 45:3-21. [PubMed: 26639665] 
14. Parmar M, et al. Using a SMALP platform to determine a sub-nm single particle cryo-EM membrane protein structure. Biochim Biophys Acta. 2017; 1860:378-383. [PubMed: 28993151]

15. Bayburt TH, Sligar SG. Membrane protein assembly into nanodiscs. FEBS Lett. 2010; 584:17211727. [PubMed: 19836392]

16. Jormakka M, et al. Molecular mechanism of energy conservation in polysulfide respiration. Nat Struct Mol Biol. 2008; 15:730-737. [PubMed: 18536726]

17. Majumder EL, King JD, Blankenship RE. Alternative Complex III from phototrophic bacteria and its electron acceptor auracyanin. Biochim Biophys Acta. 2013; 1827:1383-1391. [PubMed: 23357331]

18. Refojo PN, Teixeira M, Pereira MM. The Alternative Complex III: properties and possible mechanisms for electron transfer and energy conservation. Biochim Biophys Acta. 2012; 1817:1852-1859. [PubMed: 22609325]

19. Crofts AR. The cytochrome $b c_{1}$ complex: function in the context of structure. Annu Rev Physiol. 2004; 66:689-733. [PubMed: 14977419]

20. Sazanov LA. A giant molecular proton pump: structure and mechanism of respiratory Complex I. Nat Rev Mol Cell Biol. 2015; 16:375-388. [PubMed: 25991374]

21. Kovacs-Simon A, Titball RW, Michell SL. Lipoproteins of bacterial pathogens. Infect Immun. 2011; 79:548-561. [PubMed: 20974828]

22. Kao WC, et al. The obligate respiratory supercomplex from Actinobacteria. Biochim Biophys Acta. 2016; 1857:1705-1714. [PubMed: 27472998]

23. Graf S, et al. Rapid electron transfer within the III-IV Supercomplex in Corynebacterium glutamicum. Sci Rep. 2016; 6:34098. [PubMed: 27682138]

24. McBride MJ, Baker SA. Development of techniques to genetically manipulate members of the genera Cytophaga, Flavobacterium, Flexibacter, and Sporocytophaga. Appl Environ Microbiol. 1996; 62:3017-3022. [PubMed: 8702294]

25. Bell AJ, Frankel LK, Bricker TM. High yield non-detergent Isolation of Photosystem I-lightharvesting chlorophyll II membranes from spinach thylakoids: implications for the organization of the PSI antennae in higher plants. J Biol Chem. 2015; 290:18429-18437. [PubMed: 26055710]

26. Berry EA, Trumpower BL. Simultaneous determination of hemes $a, b$, and $c$ from pyridine hemochrome spectra. Anal Biochem. 1987; 161:1-15. [PubMed: 3578775]

27. Thomas PE, Ryan D, Levin W. An improved staining procedure for the detection of the peroxidase activity of cytochrome P-450 on sodium dodecyl sulfate polyacrylamide gels. Anal Biochem. 1976; 75:168-176. [PubMed: 822747]

28. Minghetti KC, et al. Modified, large-scale purification of the cytochrome o complex (bo-type oxidase) of Escherichia coli yields a two heme/one copper terminal oxidase with high specific activity. Biochemistry. 1992; 31:6917-6924. [PubMed: 1322173]

29. Gao X, Xin Y, Blankenship RE. Enzymatic activity of the Alternative Complex III as a menaquinol:auracyanin oxidoreductase in the electron transfer chain of Chloroflexus aurantiacus. FEBS Lett. 2009; 583:3275-3279. [PubMed: 19755122]

30. Ragan, CI., Wilson, MT., Darley-Usmar, VM., Lowe, PN. Mitochondria: A Practical Approach. IRL Press; 1987.

31. Carrell CJ, et al. Generation of novel copper sites by mutation of the axial ligand of amicyanin. Atomic resolution structures and spectroscopic properties. Biochemistry. 2007; 46:1900-1912. [PubMed: 17295442]

32. Ouyang $\mathrm{H}$, et al. Functional importance of a pair of conserved glutamic acid residues and of $\mathrm{Ca}^{2+}$ binding in the $c b b_{3}$-type oxygen reductases from Rhodobacter sphaeroides and Vibrio cholerae. Biochemistry. 2012; 51:7290-7296. [PubMed: 22913716]

33. Bowyer JR, Tierney GV, Crofts AR. Cytochrome $c_{2}$--reaction centre coupling in chromatophores of Rhodopseudomonas sphaeroides and Rhodopseudomonas capsulata. FEBS Lett. 1979; 101:207-212. [PubMed: 221250]

34. Dutton PL. Redox potentiometry: determination of midpoint potentials of oxidation-reduction components of biological electron-transfer systems. Methods Enzymol. 1978; 54:411-435. [PubMed: 732578] 
35. Fultz M, Durs R. Mediator compounds for the electrochemical study of biological redox systems: a compilation. Anal Chim Acta. 1982:140.

36. Marr CR, Benlekbir S, Rubinstein JL. Fabrication of carbon films with 500nm holes for cryo-EM with a direct detector device. J Struct Biol. 2014; 185:42-47. [PubMed: 24269484]

37. Tivol WF, Briegel A, Jensen GJ. An improved cryogen for plunge freezing. Microsc Microanal. 2008; 14:375-379. [PubMed: 18793481]

38. Suloway C, et al. Automated molecular microscopy: The new Leginon system. J Struct Biol. 2005; 151:41-60. [PubMed: 15890530]

39. Zheng SQ, et al. MotionCor2: anisotropic correction of beam-induced motion for improved cryoelectron microscopy. Nat Methods. 2017; 14:331-332. [PubMed: 28250466]

40. Rohou A, Grigorieff N. CTFFIND4: Fast and accurate defocus estimation from electron micrographs. J Struct Biol. 2015; 192:216-221. [PubMed: 26278980]

41. Scheres SHW. A bayesian view on cryo-EM structure determination. J Mol Biol. 2012; 415:406418. [PubMed: 22100448]

42. Scheres SHW. Semi-automated selection of cryo-EM particles in RELION-1.3. J Struct Biol. 2015; 189:114-122. [PubMed: 25486611]

43. Punjani A, Rubinstein JL, Fleet DJ, Brubaker M. A cryoSPARC: algorithms for rapid unsupervised cryo-EM structure determination. Nat Methods. 2017; 14:290-296. [PubMed: 28165473]

44. Pettersen EF, et al. UCSF Chimera--A visualization system for exploratory research and analysis. J Comput Chem. 2004; 25:1605-1612. [PubMed: 15264254]

45. Tsirigos KD, Peters C, Shu N, Käll L, Elofsson A. The TOPCONS web server for consensus prediction of membrane protein topology and signal peptides. Nucleic Acids Res. 2015; 43:W401W407. [PubMed: 25969446]

46. Drozdetskiy A, Cole C, Procter J, Barton GJ. JPred4: a protein secondary structure prediction server. Nucleic Acids Res. 2015; 43:W389-W394. [PubMed: 25883141]

47. Emsley P, Lohkamp B, Scott WG, Cowtan K. Features and development of Coot. Acta Cryst D. 2010; 66:486-501. [PubMed: 20383002]

48. Adams PD, et al. PHENIX : a comprehensive Python-based system for macromolecular structure solution. Acta Cryst D. 2010; 66:213-221. [PubMed: 20124702]

49. Chen VB, et al. MolProbity: all-atom structure validation for macromolecular crystallography. Acta Cryst D. 2010; 66:12-21. [PubMed: 20057044]

50. Barad BA, et al. EMRinger: side chain-directed model and map validation for 3D cryo-electron microscopy. Nat Methods. 2015; 12:943. [PubMed: 26280328]

51. Humphrey W, Dalke A, Schulten K. VMD: Visual molecular dynamics. J Mol Graphics. 1996; $14: 33-38$

52. Källberg M, et al. Template-based protein structure modeling using the RaptorX web server. Nat Protoc. 2012; 7:1511-1522. [PubMed: 22814390]

53. Madden TL, Tatusov RL, Zhang J. Applications of network BLAST server. Methods Enzymol. 1996; 266:131-141. [PubMed: 8743682]

54. Sievers F, et al. Fast, scalable generation of high-quality protein multiple sequence alignments using Clustal Omega. Mol Syst Biol. 2011:7.

55. Robert X, Gouet P. Deciphering key features in protein structures with the new ENDscript server. Nucleic Acids Res. 2014; 42:W320-W324. [PubMed: 24753421]

56. Jorgensen WL, Chandrasekhar J, Madura JD, Impey RW, Klein ML. Comparison of simple potential functions for simulating liquid water. J Chem Phys. 1983; 79:926-935.

57. Singharoy A, et al. Molecular dynamics-based refinement and validation for sub-5 A cryo-electron microscopy maps. eLife. 2016; 5:61-67.

58. Huang J, et al. CHARMM36m: an improved force field for folded and intrinsically disordered proteins. Nat Methods. 2016; 14:71-73. [PubMed: 27819658]

59. Best RB, et al. Optimization of the additive CHARMM all-atom protein force field targeting improved sampling of the backbone phi, psi and side-chain chil and chi2 dihedral angles. J Chem Theory Comput. 2012; 8:3257-3273. [PubMed: 23341755] 
60. Autenrieth F, Tajkhorshid E, Baudry J, Luthey-Schulten Z. Classical force field parameters for the heme prosthetic group of cytochrome $c$. J Comput Chem. 2004; 25:1613-1622. [PubMed: 15264255]

61. Autenrieth F, Tajkhorshid E, Klaus Schulten A, Luthey-Schulten Z. Role of Water in Transient Cytochrome $c_{2}$ Docking. J Phys Chem B. 2004; 108:20376-20387.

62. Carvalho ATP, Swart M. Correction for electronic structure investigation and parametrization of biologically relevant iron-sulfur clusters. J Chem Inf Model. 2015; 55:1508-1508. [PubMed: 26126646]

63. Carvalho ATP, Swart M. Electronic Structure Investigation and Parametrization of Biologically Relevant Iron-Sulfur Clusters. J Chem Inf Model. 2014; 54:613-620. [PubMed: 24460186]

64. Allen, MP., Tildesley, DJ. Computer Simulation of Liquids. Oxford University Press; 1987.

65. Fletcher R, Reeves CM. Function minimization by conjugate gradients. Comput J. 1964; 7:149154.

66. Sun, W., Yuan, Y-X. Optimization theory and methods: nonlinear programming. Springer; 2006. 

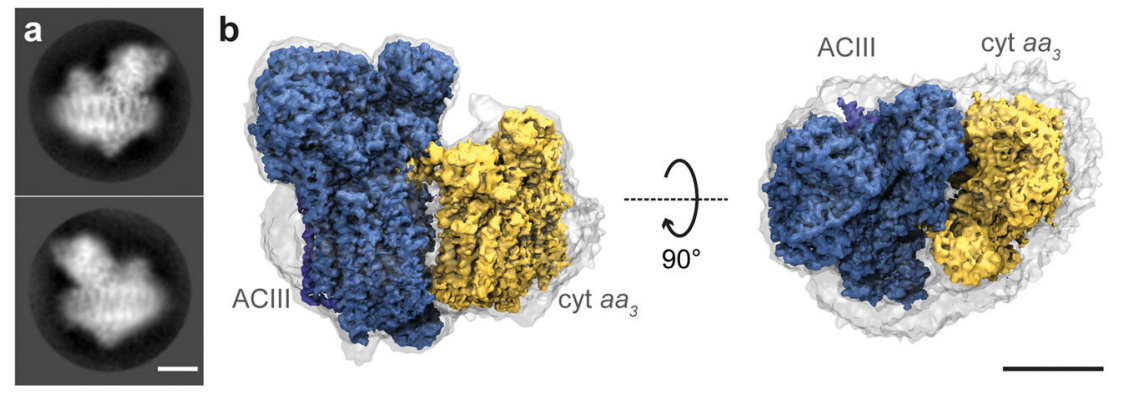

Figure 1.

Cryo-EM of ACIII-cyt aa 3 supercomplex in SMA nanodisc. a, Two representative 2-D class average images of the ACIII-cyt $a a_{3}$ supercomplex in a nanodisc. Calculation of 2-D class averages was not repeated. b, Side and top views of the ACIII-cyt aa 3 supercomplex cryoEM map. The transparent surface indicates the boundary of the nanodisc. Scale bars, $50 \AA$. 


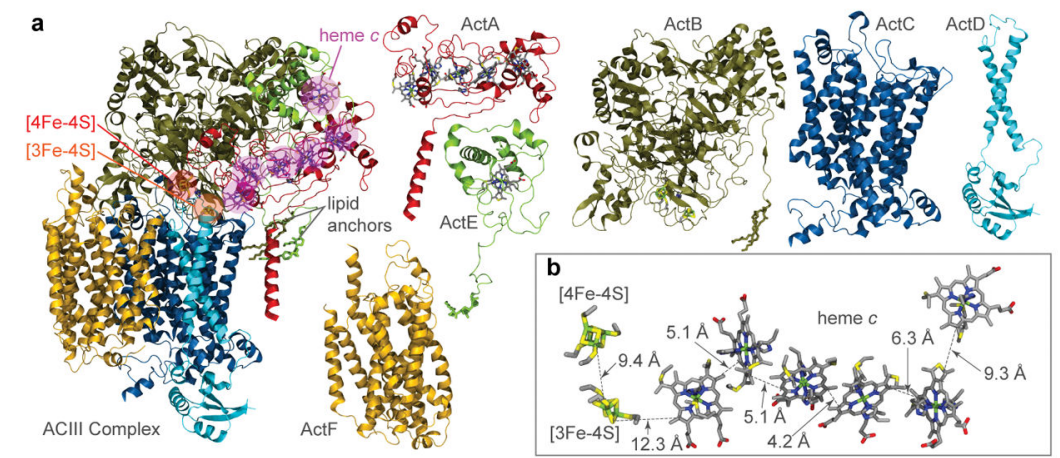

Figure 2.

Atomic model of ACIII. a, The overall structure is shown (Left) along with separate views of individual subunits. Covalently bonded cysteines are shown along with the core iron sulfur clusters (circled). Both covalently linked cysteines and axial coordinating residues are shown for hemes (circled). b, Edge-to-edge distances between cofactors. 


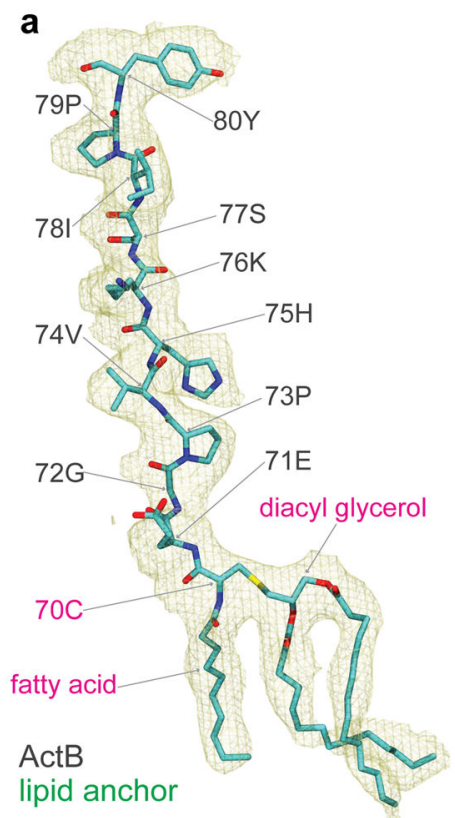

b

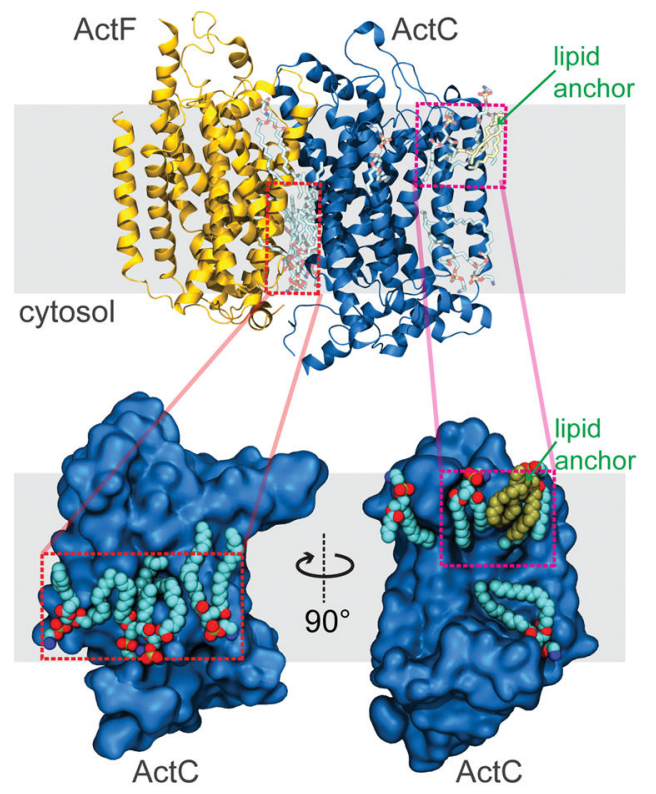

Figure 3.

Lipids in the structure of ACIII. a, Triacylated cysteine at the $\mathrm{N}$ terminus of ActB. The triacylated cysteine and its downstream ten amino acid residues are shown in the context of the experimental density map. b, Other resolved lipids near the transmembrane a-helices. Four lipid molecules are resolved at the cytoplasmic interface between ActC and ActF. Besides, two lipid molecules clustered near the triacylated cysteine from ActB, right above the proposed quinone entry pathway. 


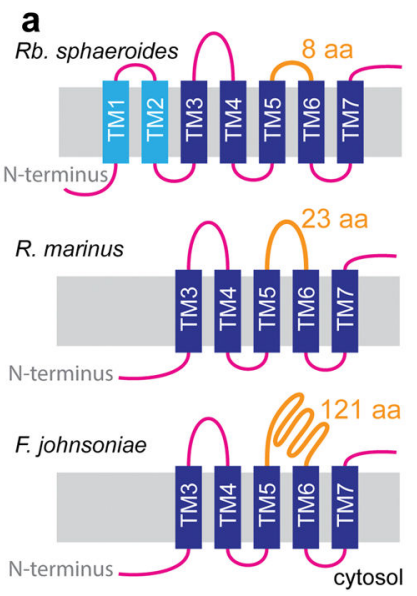

b

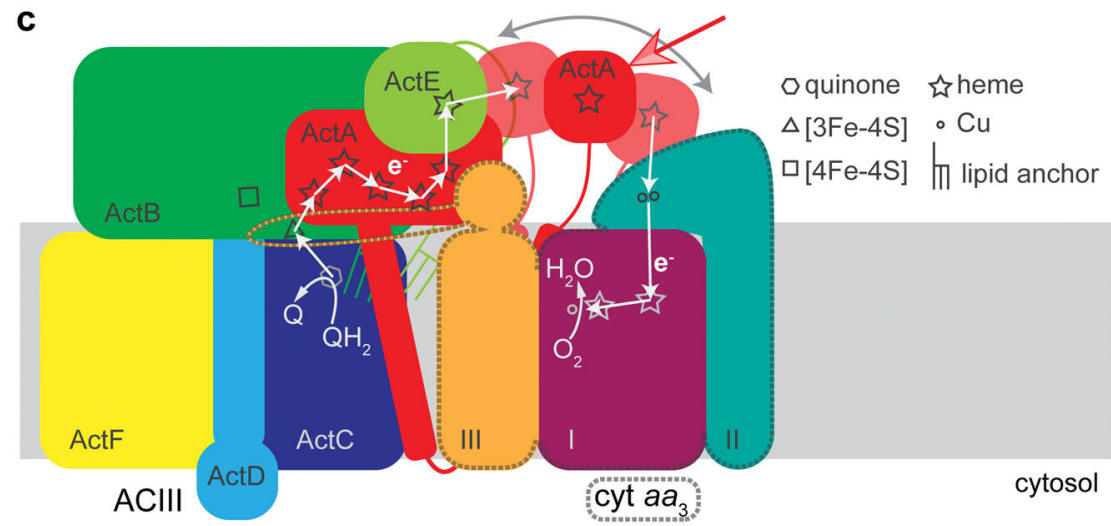

Figure 4.

ACIII-cyt $a_{3}$ supercomplex in Flavobacterium johnsoniae. a, Topology comparison of subunit III of cyt $a a_{3}$. b, Tryptophan 188 from the subunit III loop (orange) of cyt aa3 interacts with arginine 868 from the subunit B of ACIII. The surface presentation of ACIII on the right shows the binding pocket for the subunit III loop. c, Working model for electron transfer in the ACIII-cyt $a a_{3}$ supercomplex. 